\title{
Brave induction: a logical framework for learning from incomplete information
}

\author{
Chiaki Sakama $\cdot$ Katsumi Inoue
}

Received: 29 October 2008 / Revised: 3 April 2009 / Accepted: 20 April 2009 /

Published online: 12 June 2009

Springer Science+Business Media, LLC 2009

\begin{abstract}
This paper introduces a novel logical framework for concept-learning called brave induction. Brave induction uses brave inference for induction and is useful for learning from incomplete information. Brave induction is weaker than explanatory induction which is normally used in inductive logic programming, and is stronger than learning from satisfiability, a general setting of concept-learning in clausal logic. We first investigate formal properties of brave induction, then develop an algorithm for computing hypotheses in full clausal theories. Next we extend the framework to induction in nonmonotonic logic programs. We analyze computational complexity of decision problems for induction on propositional theories. Further, we provide examples of problem solving by brave induction in systems biology, requirement engineering, and multiagent negotiation.
\end{abstract}

Keywords Brave induction · Inductive logic programming · Nonmonotonic logic programming

\section{Introduction}

\subsection{Explanatory induction}

Logical foundations for induction is one of the central topics in machine learning, and different theories of induction have been proposed in the literature (Plotkin 1970; Helft 1989; De Raedt and Lavrač 1993; Muggleton 1995; Inoue 2004; Sakama 2005, for instance).

Editors: Filip Zelezny and Nada Lavrac.

C. Sakama $(\bowtie)$

Department of Computer and Communication Sciences, Wakayama University, Sakaedani, Wakayama 640-8510, Japan

e-mail: sakama@sys.wakayama-u.ac.jp

K. Inoue

National Institute of Informatics, 2-1-2 Hitotsubashi, Chiyoda-ku, Tokyo 101-8430, Japan

e-mail: ki@nii.ac.jp 
A typical induction task constructs hypotheses to explain an observation (or examples) using background knowledge. More precisely, given a first-order theory $B$ as background knowledge and a formula $O$ as an observation, a hypothesis $H$ covers $O$ under $B$ if

$$
B \wedge H \models O
$$

where $B \wedge H$ is consistent. This style of induction is called explanatory induction (Flach 1996) or learning from entailment (De Raedt 1997). It is used as a normal setting in inductive logic programming (ILP) (Muggleton and De Raedt 1994; Nienhuys-Cheng and De Wolf 1997) and is also used for induction from full clausal theories (Inoue 2004).

By the definition, explanatory induction requires that a possible solution $H$ together with $B$ logically entails $O$. In other words, $O$ is true in every model of $B \wedge H$. This condition is often too strong for building possible hypotheses, however.

Example 1.1 Suppose that there are 30 students in a class, of which 20 are European, 7 are Asian, and 3 are American. The situation is represented by background knowledge $B$ and the observation $O$ :

$$
\begin{aligned}
& B: \text { student }(1) \wedge \cdots \wedge \text { student }(30), \\
& O: \operatorname{euro}(1) \wedge \cdots \wedge \operatorname{euro}(20) \wedge \operatorname{asia}(21) \wedge \cdots \wedge \operatorname{asia}(27) \wedge u \operatorname{usa}(28) \wedge \cdots \wedge u \operatorname{sa}(30)
\end{aligned}
$$

where each number represents individual students. In this case, the following clause, saying that every student is either European, Asian, or American, appears a good hypothesis:

$$
H: \operatorname{euro}(x) \vee \operatorname{asia}(x) \vee u \operatorname{sa}(x) \leftarrow \operatorname{student}(x)
$$

Unfortunately, however, $H$ does not satisfy the relation $B \wedge H \models O$. In fact, $B \wedge H$ has many models in which $O$ is not true. An instance of such a model is:

$$
\{\text { student }(1), \ldots, \text { student }(30), \text { euro(1), .., euro(30) }\}
$$

Explanatory induction in ILP has mainly been used for learning Horn theories. When background knowledge $B$ and a hypothesis $H$ are Horn theories, the intersection of all models of $B \wedge H$ coincides with the unique minimal model (or the least model). The relation (1) then implies that $O$ is true in the least model of $B \wedge H$. On the other hand, when $B$ or $H$ contains indefinite information, $B \wedge H$ becomes a non-Horn theory which has multiple minimal models in general. In this case, an observation $O$ may be true in some minimal models of $B \wedge H$ but not every one. However, the relation (1) excludes a hypothesis $H$ due to the existence of a (minimal) model in which $O$ is not true. As a result, meaningful hypotheses might be unqualified as presented above.

\subsection{Learning from interpretation and learning from satisfiability}

Explanatory induction is used for classifying observed data and predicting unseen phenomena. By contrast, learning from interpretations (LFI) (De Raedt 1997; De Raedt and Dehaspe 1997a) seeks regularities over observed data. In LFI observations are given as interpretations, and induction seeks hypotheses which are satisfied by observations expanded by 
background knowledge. More precisely, a hypothesis $H$ covers $O$ under $B$ in LFI iff $H$ is true in the least model of $B \wedge O{ }^{1}$

Example 1.2 Consider background knowledge $B$ and the observation $O$ in Example 1.1, in which $O$ is viewed as the interpretation containing atoms appearing in $O$. Then, the hypothesis $H$ of (2) covers $O$ under $B$ in LFI. Suppose, on the other hand, that $B^{\prime}=B \cup$ \{student(31)\}. Then, $H$ does not cover $O$ under $B^{\prime}$ in LFI as the least model of $B^{\prime} \wedge O$ does not satisfy $H$.

In Example 1.2, when there is a student whose nationality is unknown, LFI does not infer the hypothesis $H$. This is because in LFI, observations are assumed to be completely specified. Thus, if complete knowledge of observations is unavailable, one should be cautious with this approach (De Raedt and Dehaspe 1997a).

Learning from satisfiability (LFS) (De Raedt 1997; De Raedt and Dehaspe 1997b) is used for concept-learning in face of incompletely specified observations. By the definition, a hypothesis $H$ covers $O$ under $B$ in LFS iff $B \wedge H$ has a model satisfying $O$.

Example 1.3 Consider again background knowledge $B$ and the observation $O$ in Example 1.1. Then, the hypothesis $H$ of (2) covers $O$ under $B$ in LFS. In Example 1.2, $H$ also covers $O$ under $B^{\prime}$ in LFS.

Thus, learning from satisfiability can induce the hypothesis $H$ of (2) under both $B$ and $B^{\prime}$. Due to its weak setting, however, the hypothesis space of LFS is generally huge. In fact, any theory $H$ becomes a possible solution of LFS as far as it is consistent with $B \wedge O$. In Example 1.1, the following hypotheses:

$$
\begin{aligned}
& H_{1}: \operatorname{euro}(x) \vee \operatorname{student}(x) \leftarrow, \\
& H_{2}: \operatorname{student}(x) \leftarrow \operatorname{euro}(x) \wedge \operatorname{asia}(x), \\
& H_{3}: \leftarrow \operatorname{asia}(x) \wedge \operatorname{usa}(x),
\end{aligned}
$$

all become solutions of LFS.

\subsection{Brave inference and cautious inference}

When $B \wedge H$ is a non-Horn theory, $B \wedge H$ has multiple minimal models in general. In this case, two different types of inferences, brave inference and cautious inference, are considered in nonmonotonic logics (McDermott 1982) and disjunctive logic programs (Eiter and Gottlob 1995). Under the minimal model semantics, a formula $F$ is a consequence of brave inference in a theory $T$ if $F$ is true in some minimal model of $T$. By contrast, $F$ is a consequence of cautious inference in $T$ if $F$ is true in every minimal model of $T$. Brave and cautious inferences are also applied to abduction in artificial intelligence. Given background knowledge $B$, an observation $O$ is explained under brave (resp. cautious) abduction if $O$ is true in some (resp. every) minimal model of a consistent theory $B \wedge H$ (Inoue and Sakama 1996; Eiter et al. 1997). Here, $H \subseteq A$ and $A$ is a set of formulas representing candidate hypotheses (called abducibles).

\footnotetext{
${ }^{1}$ This is a definition for a definite clause theory $B$ (De Raedt and Dehaspe 1997a), and its extension to nonHorn theory is discussed in Sect. 6.2.
} 
Example 1.4 Suppose background knowledge $B$ and abducibles $A$ :

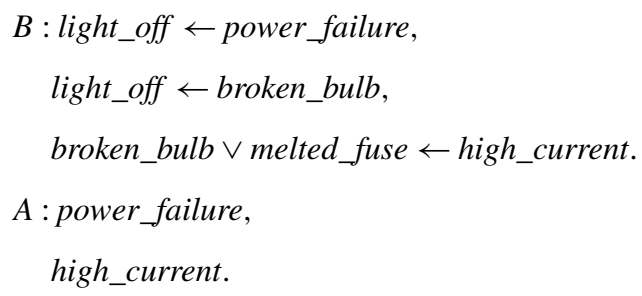

Given the observation $O=$ light_off, $E_{1}=$ power_failure is the unique (minimal) explanation in cautious abduction, while $E_{2}=$ high_current as well as $E_{1}$ are two (minimal) explanations in brave abduction.

Thus, brave inference is weaker than cautious inference, and it is especially useful for hypothetical reasoning as it can compute more hypotheses than cautious one.

\subsection{Outline of the paper}

In this paper, we apply brave inference to induction. Brave induction can induce non-Horn clauses from a full clausal theory with incomplete observations. It is weaker than explanatory induction but stronger than learning from satisfiability, thus provides a reasonable compromise between the two frameworks. Using brave induction, the hypothesis (2) becomes a solution of both $B$ of Example 1.1 and $B^{\prime}$ of Example 1.2. Brave induction is also defined for induction from nonmonotonic logic programs containing default negation. We show potential applications of brave induction for problem solving in systems biology, requirement engineering, and multiagent negotiation.

The rest of this paper is organized as follows. Section 2 introduces a logical framework of brave induction and develops a procedure for computing hypotheses. Section 3 extends the framework to induction from nonmonotonic logic programs. Section 4 analyzes computational complexity of brave induction on propositional theories. Section 5 addresses problem solving by brave induction. Section 6 discusses related issues, and Sect. 7 concludes the paper. This paper is a revised and extended version of (Sakama and Inoue 2008). Sections 4 and 5 are new in this paper. Moreover, new considerations and additional arguments are added throughout the paper.

\section{Brave induction}

\subsection{Logical framework}

We first introduce a logical framework of induction considered in this paper. A first-order language $\mathcal{L}$ consists of an alphabet and all formulas defined over it. The definition is the standard one in the literature (Nienhuys-Cheng and De Wolf 1997). For induction we use a clausal language which is a subset of $\mathcal{L}$.

A clausal theory (or simply a theory) is a finite set of clauses of the form:

$$
A_{1} \vee \cdots \vee A_{m} \vee \neg A_{m+1} \vee \cdots \vee \neg A_{n} \quad(n \geq m \geq 0)
$$


where each $A_{i}(1 \leq i \leq n)$ is an atom. Any variable in a clause is assumed to be universally quantified at the front. A clause of the above form is also written as

$$
A_{1} \vee \cdots \vee A_{m} \leftarrow A_{m+1} \wedge \cdots \wedge A_{n}
$$

$A_{1} \vee \cdots \vee A_{m}$ is the head of the clause, and $A_{m+1} \wedge \cdots \wedge A_{n}$ is the body. Given a clause $C$ of the above form, head $(C)$ represents the set $\left\{A_{1}, \ldots, A_{m}\right\}$ and $\operatorname{body}(C)$ represents the set $\left\{A_{m+1}, \ldots, A_{n}\right\}$. A clause $C$ is often identified with the set of literals $\left\{A_{1}, \ldots, A_{m}, \neg A_{m+1}, \ldots, \neg A_{n}\right\}$. A positive clause is a clause $C$ with $\operatorname{body}(C)=\emptyset$, and a negative clause is a clause $C$ with $\operatorname{head}(C)=\emptyset$. A Horn clause is a clause $C$ with $\mid$ head $(C) \mid \leq 1$. A Horn theory is a finite set of Horn clauses. A theory is identified with the conjunction of the clauses in it. A theory, a clause or an atom is ground if it contains no variable. A (ground) substitution $\theta$ replaces variables $x_{1}, \ldots, x_{k}$ occurring in a clause $C$ (resp. an atom $A$ ) to (ground) terms $t_{1}, \ldots, t_{k}$ in $C \theta$ (resp. $A \theta$ ). A clause $C$ subsumes a clause $D$ if there is a substitution $\theta$ such that $C \theta \subseteq D$. A conjunctive normal form (CNF) formula is a conjunction of disjunctions of literals, and a disjunctive normal form (DNF) formula is a disjunction of conjunctions of literals. A CNF formula or a DNF formula is ground if it contains no variable. A DNF formula $F=c_{1} \vee \cdots \vee c_{k}$ is irredundant if $F \not \equiv F^{\prime}$ for any $F^{\prime}=c_{1} \vee \cdots \vee c_{i-1} \vee c_{i+1} \vee \cdots \vee c_{k}(1 \leq i \leq k)$. A conjunction $C$ of ground atoms is identified with the set of ground atoms in $C$. Given a literal $L$, pred $(L)$ represents the predicate of $L$, and term $(L)$ and const $(L)$ represent the sets of terms and constants in $L$, respectively.

The domain of a theory $T$ is given as the Herbrand universe $H U$ and an interpretation of $T$ is defined as a subset of the Herbrand base $H B$. An interpretation $I$ satisfies a ground clause (3) if $\left\{A_{m+1}, \ldots, A_{n}\right\} \subseteq I$ implies $\left\{A_{1}, \ldots, A_{m}\right\} \cap I \neq \emptyset$. An interpretation $I$ satisfies a non-ground clause $C$ if $I$ satisfies every ground instance of $C$. An interpretation $I$ satisfies a theory $T$ if $I$ satisfies every clause in $T$. An interpretation $I$ is a model of $T$ if $I$ satisfies $T$. $\operatorname{Mod}(T)$ represents the set of all models of $T$. A model $M \in \operatorname{Mod}(T)$ is minimal if $N \subseteq M$ implies $M \subseteq N$ for any $N \in \operatorname{Mod}(T)$. The set of minimal models of $T$ is written as $M M(T)$. A theory $T$ entails a formula $F$ (written as $T \models F$ ) if $F$ is true in any $I \in \operatorname{Mod}(T)$. A theory $T$ is consistent if $\operatorname{Mod}(T) \neq \emptyset$; otherwise, $T$ is inconsistent.

Let $B, O$ and $H$ be all clausal theories, where $B, O$, and $H$ are respectively called a background knowledge, an observation, and a hypothesis. Here, $B, O$, and $H$ are sets of clauses, but each set is identified with the conjunction of clauses included in the set as usual. We assume that $B, O$ and $H$ have the same $H U$ and $H B$. Given $B$ and $O$, explanatory induction construct $H$ to explain $O$ under $B$. Formally, a hypothesis $H$ covers $O$ under $B$ if

$$
B \wedge H \models O
$$

where $B \wedge H$ is consistent. $H$ is called a solution of explanatory induction.

Two new frameworks of induction are introduced next.

Definition 2.1 (Brave and cautious induction) Let $B$ be background knowledge and $O$ an observation. A hypothesis $H$ covers $O$ under $B$ in brave induction if $B \wedge H$ has a minimal model satisfying $O$. In this case, $H$ is called a solution of brave induction.

By contrast, $H$ covers $O$ under $B$ in cautious induction if $B \wedge H$ is consistent and every minimal model of $B \wedge H$ satisfies $O$. In this case, $H$ is called a solution of cautious induction. 
The names of brave and cautious induction are taken from brave and cautious inferences. Under the minimal model semantics, a formula $F$ is a consequence of cautious inference in a theory $T$ if it is true in every minimal model of $T$, while $F$ is a consequence of brave inference in $T$ if $F$ is true in some minimal model of $T .^{2}$ When a theory contains indefinite or incomplete information, brave inference infers more results than cautious inference in general. Brave and cautious inferences have been used in different reasoning tasks of deduction and abduction in artificial intelligence. It is thereby natural to apply these inferences to induction from non-Horn theories containing indefinite or incomplete information.

Relations between explanatory induction, cautious induction, and brave induction are as follows.

Proposition 2.1 (Relation among solutions) Let $B$ be background knowledge and $O$ an observation.

1. If $H$ is a solution of explanatory induction, $H$ is a solution of cautious induction. The converse implication also holds when $O$ is a set of positive clauses.

2. If $H$ is a solution of cautious induction, $H$ is a solution of brave induction. The converse implication also holds when $B \wedge H$ is a Horn theory.

Proof (1) The if-part is obvious by the definition. To see the converse, if $H$ is a solution of cautious induction, any positive clause $C$ in $O$ is satisfied in every minimal model of a consistent theory $B \wedge H$. Then, $C$ is satisfied in every model of a consistent theory $B \wedge H$. (2) The if-part is obvious by the definition. When $B \wedge H$ is a Horn theory, it has a unique minimal model. Hence, the result holds.

The converse implication of Proposition 2.1(1) does not hold in general when $O$ contains a clause $C$ with $\operatorname{body}(C) \neq \emptyset$.

Example 2.1 Let $B=\{p(a) \vee q(a)\}$ and $O=\{\neg p(a)\}$. Then, $H=\{q(a)\}$ is a solution of cautious induction, but $H$ is not a solution of explanatory induction because $B \wedge H$ has a model in which $p(a)$ is true.

Proposition 2.2 (Existence of solutions) Let $B$ be background knowledge and $O$ an observation. Then, the following four conditions are equivalent.

1. $B \wedge O$ is consistent.

2. Brave induction has a solution.

3. Cautious induction has a solution.

4. Explanatory induction has a solution.

Proof We prove (1) $\Leftrightarrow(2)$, but (1) $\Leftrightarrow$ (3) and (1) $\Leftrightarrow$ (4) are proved in the same manner. If brave induction has a solution $H, B \wedge H$ has a minimal model satisfying $O$. Then, $B \wedge O$ is consistent. To see the converse, suppose that brave induction has no solution. Then, for any $H$ no minimal model of $B \wedge H$ satisfies $O$. This implies that for any $H$ no minimal model exists for $B \wedge H \wedge O$, so $B \wedge H \wedge O$ is inconsistent for any $H .{ }^{3}$ Putting $H=\emptyset, B \wedge O$ is inconsistent. Hence, the result holds.

\footnotetext{
${ }^{2}$ Brave and cautious inferences are also called credulous and skeptical inferences, respectively.

${ }^{3}$ Every consistent clausal theory has a minimal model (Bossu and Siegel 1985).
} 
Corollary 2.3 (Necessary condition of solutions) Let $B$ be background knowledge and $O$ an observation. If $H$ is a solution of brave induction, $B \wedge H \wedge O$ is consistent. The same condition holds for cautious and explanatory induction.

We later provide another necessary and sufficient condition for brave induction in Proposition 2.7.

Next we compare properties of brave and cautious induction. In what follows, $B, O$, and $H$ represent background knowledge, an observation, and a hypothesis, respectively.

Proposition 2.4 (Conjunction of solutions) The fact that both $H_{1}$ and $H_{2}$ are solutions of brave induction does not imply that $\mathrm{H}_{1} \wedge \mathrm{H}_{2}$ is a solution of brave induction. This is also the case for cautious induction.

The above property also holds for explanatory induction (De Raedt and Dehaspe 1997b). ${ }^{4}$

Example 2.2 Let $B=\{p(a) \leftarrow\}$ and $O=\{q(a) \vee r(a) \leftarrow, \leftarrow q(a) \wedge r(a)\}$. Then, both $H_{1}=\{q(x) \leftarrow p(x)\}$ and $H_{2}=\{r(x) \leftarrow p(x)\}$ cover $O$ under $B$ in brave or cautious induction, but $H_{1} \wedge H_{2}$ does not.

Proposition 2.5 (Disjunction of solutions) If $H_{1}$ and $H_{2}$ are solutions of brave induction, so is $H_{1} \vee H_{2}$. This is also the case for cautious and explanatory induction.

Proof By $B \wedge\left(H_{1} \vee H_{2}\right) \equiv\left(B \wedge H_{1}\right) \vee\left(B \wedge H_{2}\right)$, if both $B \wedge H_{1}$ and $B \wedge H_{2}$ have a minimal model satisfying $O$, so does $B \wedge\left(H_{1} \vee H_{2}\right)$. Moreover, if $O$ is satisfied in every (minimal) model of $B \wedge H_{1}$ and $O$ is satisfied in every (minimal) model of $B \wedge H_{2}, O$ is also satisfied in every (minimal) model of $\left(B \wedge H_{1}\right) \vee\left(B \wedge H_{2}\right)$.

Proposition 2.6 (Conjunctions of observations) The fact that $\mathrm{H}$ covers both $\mathrm{O}_{1}$ and $\mathrm{O}_{2}$ under $B$ implies that $H$ covers $O_{1} \wedge O_{2}$ under $B$ in cautious and explanatory induction. But this is not the case for brave induction.

Proof If $O_{1}$ and $O_{2}$ are satisfied in every (minimal) model of $B \wedge H$, so does $O_{1} \wedge O_{2}$. A counter-example for brave induction is shown in Example 2.3 below.

Example 2.3 Let $B=\{p(x) \vee q(x) \leftarrow r(x), s(a) \leftarrow\}, O_{1}=\{p(a)\}$, and $O_{2}=\{q(a)\}$. Then, $H=\{r(x) \leftarrow s(x)\}$ covers both $O_{1}$ and $O_{2}$ under $B$ in brave induction, but $H$ does not cover $O_{1} \wedge O_{2}$ under $B$.

Proposition 2.6 provides a property that distinguishes brave induction from cautious and explanatory induction. This property implies that given a series of observations, brave induction is not adapted for performing incremental computation of candidate hypotheses in general. Such an incremental computation is done in brave induction for hypotheses that are also solutions of cautious or explanatory induction.

Table 1 summarizes comparison of properties between brave, cautious and explanatory induction. By the table, we can observe that cautious induction and explanatory induction share similar properties. In fact, the difference between cautious and explanatory induction

\footnotetext{
${ }^{4}$ This property is also called nonmonotonicity of induction.
} 
Table 1 Comparison of properties

Brave Ind.

Conjunction of solutions

Disjunction of solutions

Conjunction of observations
Cautious Ind.

Explanatory Ind.

$\times$

$\times$ $\bigcirc$ $\bigcirc \quad 0$

○

$\times$

arises only when $O$ contains non-positive clause (Proposition 2.1(1)). In many induction tasks, however, observations are usually given as a set of positive clauses or ground facts. On the other hand, brave induction may have solutions different from those of cautious or explanatory induction when $B$ or $H$ contains disjunctive clauses (Proposition 2.1(2)). Then, we are interested in computing possible solutions of brave induction under background knowledge containing indefinite information, or computing solutions containing disjunctive information.

When $B$ has a minimal model satisfying $O, O$ is inferred by brave inference from $B$. In this case, $H=$ true covers $O$, which is a trivial and uninteresting solution. The problem of our interest is the case in which $B$ has no minimal model satisfying $O$. When a clausal theory $B$ has no minimal model satisfying $O, \neg O$ is derived from $B$ under the generalized closed world assumption (GCWA) (Minker 1982).

Example 2.4 Let $B=\{p(a) \vee q(b)\}$ be background knowledge which has two minimal models $\{p(a)\}$ and $\{q(b)\}$. Then, $H=$ true covers the observation $O_{1}=\{p(a)\}$ under $B$ in brave induction, while $H$ does not cover the observation $O_{2}=\{p(b)\}$. In this case, the GCWA derives $\neg p(b)$ but does not derive $\neg p(a)$.

It is worth noting that explanatory induction in Horn theories assumes that the background Horn theory $B$ has no minimal model satisfying $O .^{5}$ In this case, $\neg O$ is derived from $B$ under the closed world assumption (CWA) (Reiter 1978). Thus, brave induction in non-Horn theories is considered a natural extension of explanatory induction in Horn theories. In the next subsection, we develop an algorithm for computing brave induction.

\subsection{Computation}

In this section, we develop an algorithm for computing brave induction. We first characterizes the brave induction problem.

Proposition 2.7 (Necessary and sufficient condition for brave induction) Let $B$ be background knowledge, $H$ a hypothesis, and $O$ an observation. Then, $B \wedge H$ has a minimal model satisfying $O$ iff there is a disjunction $F$ of ground atoms such that $B \wedge H \models O \vee F$ and $B \wedge H \not \models F$.

Proof $(\rightarrow)$ Suppose that $B \wedge H$ has a minimal model $M$ such that $M \models O$. Consider a disjunction $F$ of ground atoms satisfying (i) $M \not \models F$ and (ii) $N \models F$ for any $N \in M M(B \wedge$

\footnotetext{
${ }^{5}$ The condition is called prior necessity (Muggleton and De Raedt 1994).

${ }^{6}$ Related results are shown in (Gelfond et al. 1989, Theorem 4.5) in the context of circumscription, and in (Inoue 2002, Corollary 3.5) in terms of abduction.
} 
$H$ ) such that $N \not \models O$. Such $F$ is constructed by picking up ground atoms from each $N \backslash M$. Then, $B \wedge H \models O \vee F$ holds. As $M \not \models F, B \wedge H \not \models F$.

$(\leftarrow)$ Suppose that $B \wedge H \models O \vee F$ holds for a disjunction $F$ of ground atoms and $B \wedge H \not \models F$. If $B \wedge H$ has no minimal model satisfying $O, B \wedge H \models O \vee F$ implies $B \wedge H \models$ $F$. This contradicts the assumption that $B \wedge H \not \forall F$.

Throughout the section, the following conditions are assumed on the syntax of observations and hypotheses.

1. An observation $O$ is a finite set of ground atoms.

2. A hypothesis $H$ is a finite clausal theory such that each clause has the non-empty head.

The first condition is assumed as the normal problem setting in ILP (Nienhuys-Cheng and De Wolf 1997). The second condition is also natural with the following reason. When $B$ has no minimal model satisfying $O$, we introduce $H$ to $B$ to get a minimal model satisfying $O$. However, introducing negative clauses to $B$ has an effect of eliminating minimal models of $B$ but does not contribute to obtaining a new minimal model. So the exclusion of negative clauses in $H$ is not a strong restriction.

The procedure for computing brave induction consists of four steps.

\section{Step 1: Computing ground hypotheses}

By Proposition 2.7, a solution of brave induction is obtained by computing $H$ satisfying

$$
B \wedge H \models O \vee F
$$

and

$$
B \wedge H \not=F \text {. }
$$

By (5), it holds that

$$
B \wedge \neg O \models \neg H \vee F .
$$

$\neg H \vee F$ is thus obtained by deduction from $B \wedge \neg O$. This technique is inverse entailment that was originally proposed by Muggleton for induction in Horn theories (Muggleton 1995), and was later extended by Inoue to full clausal theories (Inoue 2004).

As $H$ is a clausal theory, put

$$
H=\left(\Sigma_{1} \leftarrow \Gamma_{1}\right) \wedge \cdots \wedge\left(\Sigma_{k} \leftarrow \Gamma_{k}\right)
$$

where $\Sigma_{i}(i=1, \ldots, k)$ is a disjunction of atoms and $\Gamma_{i}(i=1, \ldots, k)$ is a conjunction of atoms. It then becomes

$$
\neg H=\left(\neg \Sigma_{1} \wedge \Gamma_{1}\right) \vee \cdots \vee\left(\neg \Sigma_{k} \wedge \Gamma_{k}\right)
$$

Since $F$ is a disjunction of ground atoms, every formula $\neg H \vee F$ in (7) is a disjunctive normal form. From $B \wedge \neg O$, a number of DNF formulas could be deduced. Among them, we take DNF formulas obtained as follows.

Definition 2.2 (Prime clause, prime CNF) A ground clause $C$ is called a prime clause with respect to a theory $T$ if $T \models C$ but $T \not C^{\prime}$ for any $C^{\prime} \subset C$. A prime $C N F$ formula with respect to $T$ is a conjunction of prime clauses with respect to $T$. 
First, compute prime $\mathrm{CNF}$ formulas with respect to $B \wedge \neg O$. Prime $\mathrm{CNF}$ formulas are computed by a system of consequence-finding such as (Inoue 1992). Second, given a prime CNF formula $c_{1} \wedge \cdots \wedge c_{k}$, produce an irredundant DNF formula $d_{1} \vee \cdots \vee d_{l}$ where $d_{i}(1 \leq$ $i \leq l)$ contains a literal from each $c_{j}(1 \leq j \leq k)$. Then,

$$
B \wedge \neg O \models d_{1} \vee \cdots \vee d_{l}
$$

holds, and we identify the DNF formula $\neg H \vee F$ of (7) with $d_{1} \vee \cdots \vee d_{l}$. After deriving such a ground DNF formula, the next task is to separate $\neg H$ and $F$ in $d_{1} \vee \cdots \vee d_{l}$. This is simply done as follows. By the assumption, $\Sigma_{i}$ in $H$ is non-empty, so that $\neg H$ is a DNF formula in which each disjunct $\neg \Sigma_{i} \wedge \Gamma_{i}$ of (9) contains at least one negative literal. Thus, from the DNF formula $d_{1} \vee \cdots \vee d_{l}, \neg H$ is extracted by selecting disjuncts containing negative literals. Hence, $H$ is obtained as a ground clausal theory.

\section{Step 2: Generalization}

As $H$ is a clausal theory containing no variable, we generalize $H$ in the next step. For this purpose, we use Plotkin's least generalization under subsumption (LGS) (Plotkin 1970).

Definition 2.3 (Comparable) Clauses $C_{1}, \ldots, C_{k}$ are comparable if there is a predicate appearing in every head $\left(C_{1}\right), \ldots$, head $\left(C_{k}\right)$.

Let $H$ be a clausal theory obtained by Step 1 . Then, $H$ is partitioned into

$$
H=H_{1} \wedge \cdots \wedge H_{n}
$$

where each $H_{i}(1 \leq i \leq n)$ is a conjunction of comparable clauses. ${ }^{7}$ Next, the LGS of each $H_{i}$ is computed and collected as

$$
\lg s(H)=\lg s\left(H_{1}\right) \wedge \cdots \wedge \lg s\left(H_{n}\right)
$$

where $\operatorname{lgs}\left(H_{i}\right)$ represents the result of LGS of $H_{i}$. Note that since each $H_{i}$ is a set of comparable clauses, $\lg s\left(H_{i}\right)$ is a clause with the non-empty head.

\section{Step 3: Constructing a weak form of hypotheses}

We next consider a method of constructing a weaker hypothesis for brave induction. For each clause $\lg s\left(H_{1}\right), \ldots, \lg s\left(H_{n}\right)$ of $(11)$, take the greatest specialization under implication (GSI) (Nienhuys-Cheng and De Wolf 1997). The GSI of any finite set of clauses exists and is computed by taking a disjunction as

$$
\operatorname{gsi}\left(\operatorname{lgs}\left(H_{1}\right), \ldots, \lg s\left(H_{n}\right)\right)=\lg s\left(H_{1}\right) \vee \cdots \vee \lg s\left(H_{n}\right) .
$$

By $\lg s\left(H_{i}\right) \models g s i\left(\lg s\left(H_{1}\right), \ldots, \lg s\left(H_{n}\right)\right)$ for $i=1, \ldots, n$, the GSI (12) provides a formula which is weaker than each $\lg s\left(H_{i}\right)$.

\section{Step 4: Optimization}

Hypotheses computed in the above steps generally contain clauses or atoms that are useless or have no direct connection to explaining the observation. In this step, hypotheses are optimized to extract meaningful information.

\footnotetext{
${ }^{7}$ A clause $C$ could be included in different $H_{i}$ and $H_{j}(i \neq j)$ if $C$ is comparable to clauses in both $H_{i}$ and $H_{j}$. 


\section{Procedure: BRAIN}

Input : the background knowledge $B$ and an observation $O$;

Output : hypotheses $H^{\wedge}$ and $H^{\vee}$.

Step 1 : Compute ground and irredundant DNF formulas $\neg H \vee F$ from $B \wedge \neg O$, and extract $\neg H$ from $\neg H \vee F$.

Step 2 : Compute the least generalization under subsumption $\lg s(H)$.

Step 3 : Compute the greatest specialization under implication $g s i\left(\operatorname{lgs}\left(H_{1}\right), \ldots, \operatorname{lgs}\left(H_{n}\right)\right)$.

Step 4 : Produce $l g s^{*}\left(H_{i}\right)$ by reduction, and compute $H^{\wedge}$ and $H^{\vee}$.

Fig. 1 An algorithm for brave induction

Definition 2.4 (Isolated) Two atoms $A_{1}$ and $A_{2}$ are linked if term $\left(A_{1}\right) \cap \operatorname{term}\left(A_{2}\right) \neq \emptyset$. Given a clause $C$ with the non-empty body, an atom $A$ is isolated in $C$ if there is no atom $A^{\prime}(\neq A)$ in $C$ such that $A^{\prime}$ and $A$ are linked.

Example 2.5 Given $C=(p(x) \leftarrow q(x, y), r(y), s(z))$, the atom $s(z)$ is isolated in $C$.

Optimization is done in two steps.

1. Remove any isolated atom from the body of any clause $\lg s\left(H_{i}\right)(1 \leq i \leq n)$.

2. Remove any clause $\lg s\left(H_{i}\right)(1 \leq i \leq n)$ that is subsumed by another clause $\lg s\left(H_{j}\right)(1 \leq$ $j \leq n)$.

The result of such reduction on $\operatorname{lgs}\left(H_{i}\right)$ is denoted by $\operatorname{lgs}{ }^{*}\left(H_{i}\right)$. When $B \wedge \operatorname{lgs}\left(H_{i}\right)$ is consistent, the reduction is performed as far as $B \wedge \operatorname{lgs}^{*}\left(H_{i}\right)$ is consistent. Finally, put

$$
H^{\wedge}=\bigwedge_{i} \lg s^{*}\left(H_{i}\right)
$$

if $B \wedge \bigwedge_{i} \operatorname{lgs}^{*}\left(H_{i}\right) \wedge \neg F$ is consistent for a disjunction $F$ of ground atoms computed by Step 1. On the other hand, put

$$
H^{\vee}=\bigvee_{i} \operatorname{lgs}^{*}\left(H_{i}\right)
$$

if there is a disjunction $F^{\prime}$ of ground atoms such that $B \wedge \bigvee_{i} \operatorname{lgs}^{*}\left(H_{i}\right) \wedge \neg F^{\prime}$ is consistent and $B \wedge \bigvee_{i} \lg s^{*}\left(H_{i}\right) \wedge \neg O \wedge \neg F^{\prime}$ is inconsistent.

The algorithm (called BRAIN) for computing hypotheses is summarized in Fig. 1.8

Now we show that BRAIN computes a solution for brave induction.

Theorem 2.8 (Soundness) Let $H^{\wedge}$ and $H^{\vee}$ be clausal theories obtained by BRAIN. Then, $H^{\wedge}$ and $H^{\vee}$ are solutions of brave induction.

Proof Step 1 computes a ground clausal theory $H$ satisfying $B \wedge H \models O \vee F$. In Step 2, $\lg s\left(H_{i}\right) \models H_{i}$ holds for each $H_{i}$ of (10). Then, $\lg s(H) \models H$ and $B \wedge \lg s(H) \models B \wedge H$. So $B \wedge \lg s(H) \models O \vee F$. In Step 4, it holds that $\lg ^{*}\left(H_{1}\right) \wedge \cdots \wedge \operatorname{lgg}^{*}\left(H_{n}\right) \models \lg s\left(H_{1}\right) \wedge$ $\cdots \wedge \lg s\left(H_{n}\right)$. Then, $\bigwedge_{i} \lg s^{*}\left(H_{i}\right) \models \lg s(H)$ and $B \wedge \bigwedge_{i} \lg s^{*}\left(H_{i}\right) \models B \wedge \lg s(H)$. So $B \wedge$ $\bigwedge_{i} \operatorname{lgs}^{*}\left(H_{i}\right) \models O \vee F$, and $\bigwedge_{i} \lg s^{*}\left(H_{i}\right)$ satisfies the relation (5). If $B \wedge \bigwedge_{i} \lg s^{*}\left(H_{i}\right) \wedge \neg F$ is

\footnotetext{
${ }^{8}$ BRAIN is named after BRAve INduction.
} 
consistent for a disjunction $F$ of ground atoms computed by Step 1 , then $B \wedge \bigwedge_{i} \operatorname{lgs}^{*}\left(H_{i}\right) \not \forall$ $F$ and $\bigwedge_{i} l g s^{*}\left(H_{i}\right)$ satisfies the relation (6). Hence, $B \wedge H^{\wedge}$ has a minimal model satisfying $O$ (Proposition 2.7).

Next, suppose $g \operatorname{si}\left(\lg s\left(H_{1}\right), \ldots, \lg s\left(H_{n}\right)\right)$ in Step 3 is optimized as $\bigvee_{i} \operatorname{lgs}^{*}\left(H_{i}\right)$ in Step 4. If $B \wedge \bigvee_{i} \operatorname{lgs}^{*}\left(H_{i}\right) \wedge \neg O \wedge \neg F^{\prime}$ is inconsistent for a disjunction $F^{\prime}$ of ground atoms, then $B \wedge \bigvee_{i} \operatorname{lgs}^{*}\left(H_{i}\right) \models O \vee F^{\prime}$. Thus, $\bigvee_{i} l g s^{*}\left(H_{i}\right)$ satisfies the relation (5). Moreover, if $B \wedge$ $\bigvee_{i} \lg ^{*}\left(H_{i}\right) \wedge \neg F^{\prime}$ is consistent, $B \wedge \bigwedge_{i} \lg s^{*}\left(H_{i}\right) \not F^{\prime}$. Then, $\bigwedge_{i} \lg s^{*}\left(H_{i}\right)$ satisfies the relation (6). Hence, $B \wedge H^{\vee}$ has a minimal model satisfying $O$ (Proposition 2.7).

Note that BRAIN is not complete with respect to solutions of brave induction. This is because we reduce seemingly useless hypotheses in the optimization phase of Step 4 . We do not consider the incompleteness of the algorithm a serious flaw, however. This is because there may exist possibly infinite solutions for explaining observations in general, and it seems meaningless to guarantee the completeness for computing tons of useless hypotheses. We select candidate solutions to reduce the hypothesis space at the cost of giving up completeness.

Example 2.6 Consider background knowledge $B$ and the observation $O$ :

$$
\begin{aligned}
& B: \text { teacher }(0) \wedge \text { student }(1) \wedge \cdots \wedge \text { student }(30), \\
& O: \text { euro }(1) \wedge \cdots \wedge \text { euro }(20) \wedge \operatorname{asia}(21) \wedge \cdots \wedge \operatorname{asia}(27) \wedge u \operatorname{usa}(28) \wedge \cdots \wedge u s a(30) .
\end{aligned}
$$

BRAIN computes candidate hypotheses as follows.

(Step 1) $B \wedge \neg O$ entails the prime clauses:

teacher $(0)$, student $(1), \cdots$, student $(30)$,

$\neg$ euro $(1) \vee \cdots \vee \neg$ euro $(20) \vee \neg$ asia $(21) \vee \cdots \vee \neg \operatorname{asia}(27) \vee \neg$ usa(28) $\vee \cdots \vee \neg$ usa(30).

Taking the conjunction of them, the prime CNF formula:

$$
\begin{aligned}
& \text { teacher }(0) \wedge \text { student }(1) \wedge \cdots \wedge \text { student }(30) \\
& \wedge(\neg \text { euro }(1) \vee \cdots \vee \neg \text { euro }(20) \vee \neg \operatorname{asia}(21) \vee \cdots \vee \neg \operatorname{asia}(27) \vee \neg \text { usa }(28) \vee \cdots \\
& \quad \vee \neg \text { usa }(30)),
\end{aligned}
$$

which is equivalent to $B \wedge \neg O$, is computed.

Next, an irredundant DNF formula $\neg H_{1} \vee \neg H_{2} \vee \neg H_{3} \vee F$ is obtained where

$$
\begin{aligned}
\neg H_{1} & =(B \wedge \neg \text { euro }(1)) \vee \cdots \vee(B \wedge \neg \text { euro }(20)), \\
\neg H_{2} & =(B \wedge \neg \text { asia }(21)) \vee \cdots \vee(B \wedge \neg \text { asia }(27)), \\
\neg H_{3} & =(B \wedge \neg \text { usa }(28)) \vee \cdots \vee(B \wedge \neg \text { usa }(30)), \\
F & =\text { false. }
\end{aligned}
$$

By this, ground hypotheses:

$$
\begin{aligned}
& H_{1}=(\neg B \vee \text { euro }(1)) \wedge \cdots \wedge(\neg B \vee \operatorname{euro}(20)), \\
& H_{2}=(\neg B \vee \operatorname{asia}(21)) \wedge \cdots \wedge(\neg B \vee \operatorname{asia}(27)), \\
& H_{3}=(\neg B \vee u s a(28)) \wedge \cdots \wedge(\neg B \vee u s a(30))
\end{aligned}
$$

are obtained. 
(Step 2) The LGS of each $H_{i}$ becomes

$$
\begin{aligned}
& \operatorname{lgs}\left(H_{1}\right)=\neg \text { teacher }(0) \vee \neg \text { student }(x) \vee \operatorname{euro}(x), \\
& \operatorname{lgs}\left(H_{2}\right)=\neg \text { teacher }(0) \vee \neg \operatorname{student}(y) \vee \operatorname{asia}(y), \\
& \operatorname{lgs}\left(H_{3}\right)=\neg \text { teacher }(0) \vee \neg \operatorname{student}(z) \vee u \operatorname{sa}(z) .
\end{aligned}
$$

Then, $\lg s(H)=\lg s\left(H_{1}\right) \wedge \lg s\left(H_{2}\right) \wedge \lg s\left(H_{3}\right)$.

(Step 3) By each $\lg s\left(H_{i}\right)$, the greatest specialization becomes

$$
\begin{aligned}
& \operatorname{gsi}\left(\operatorname{lgs}\left(H_{1}\right), \ldots, \operatorname{lgs}\left(H_{n}\right)\right)=\operatorname{lgs}\left(H_{1}\right) \vee \lg s\left(H_{2}\right) \vee \operatorname{lgs}\left(H_{3}\right) \\
& \quad=\neg \text { teacher }(0) \vee \neg \operatorname{student}(x) \vee \operatorname{euro}(x) \vee \operatorname{asia}(x) \vee u s a(x) .
\end{aligned}
$$

(Step 4) The atom teacher $(0)$ is isolated in each $\operatorname{lgs}\left(H_{i}\right)(i=1,2,3)$, so it is removed from the body of each clause. Since $B \wedge \bigwedge_{i} l g s^{*}\left(H_{i}\right) \wedge \neg F$ is consistent, $H^{\wedge}$ becomes

$$
(\operatorname{euro}(x) \leftarrow \operatorname{student}(x)) \wedge(\operatorname{asia}(x) \leftarrow \operatorname{student}(x)) \wedge(u \operatorname{sa}(x) \leftarrow \operatorname{student}(x)) .
$$

On the other hand, for the disjunction $F^{\prime}$ of ground atoms:

$$
\begin{aligned}
F^{\prime}= & (\operatorname{asia}(1) \vee u s a(1)) \vee \cdots \vee(\operatorname{asia}(20) \vee u s a(20)) \\
& \vee(\operatorname{euro}(21) \vee u s a(21)) \vee \cdots \vee(\text { euro }(27) \vee u s a(27)) \\
& \vee(\operatorname{asia}(28) \vee \operatorname{euro}(28)) \vee \cdots \vee(\operatorname{asia}(30) \vee \operatorname{euro}(30)),
\end{aligned}
$$

$B \wedge \bigvee_{i} \lg s^{*}\left(H_{i}\right) \wedge \neg F^{\prime}$ is consistent and $B \wedge \bigvee_{i} \lg s^{*}\left(H_{i}\right) \wedge \neg O \wedge \neg F^{\prime}$ is inconsistent.

Then, $H^{\vee}$ becomes

$$
\operatorname{euro}(x) \vee \operatorname{asia}(x) \vee u s a(x) \leftarrow \operatorname{student}(x) .
$$

As a result, $H^{\wedge}$ and $H^{\vee}$ become two solutions of brave induction.

Note that if there are negative clauses

$$
\begin{aligned}
& \leftarrow \operatorname{euro}(x) \wedge \operatorname{asia}(x), \\
& \leftarrow \operatorname{euro}(x) \wedge \operatorname{usa}(x), \\
& \leftarrow \operatorname{asia}(x) \wedge u \operatorname{sa}(x)
\end{aligned}
$$

in $B, B \wedge \bigwedge_{i} l g s^{*}\left(H_{i}\right)$ is inconsistent. In this case, $H^{\wedge}$ is not a solution of brave induction, while $H^{\vee}$ is still a solution.

Solutions of cautious induction are computed as a special case of BRAIN.

Corollary 2.9 (Computing cautious induction) Let $H^{\wedge}$ be clausal theories obtained by BRAIN. If $F=$ false in Step $1, H^{\wedge}$ is a solution of cautious induction.

Proof When $F=$ false, BRAIN computes $H$ satisfying $B \wedge H \models O$ (5) and $B \wedge H \not$ false (6). Then, $H$ is a solution of explanatory induction. Since $O$ is a set of ground atoms, solutions of explanatory induction coincide with those of cautious induction (Proposition 2.1(1)). Hence, the result holds.

In Example 2.6, $H^{\wedge}$ also becomes a solution of cautious induction. 


\section{Brave induction in nonmonotonic logic programming}

As presented in Sect. 2, brave induction is useful for learning theories with indefinite or incomplete information. Incomplete information is also represented as default rule in logic programming. In this section, we consider brave induction in nonmonotonic logic programs.

\subsection{Answer set programming}

Answer set programming (ASP) (Lifschitz 2002) represents incomplete knowledge in a logic program and realizes nonmonotonic default reasoning. In ASP a logic program is described by an extended disjunctive program (EDP). An EDP (or simply a program) is a set of rules of the form:

$$
L_{1} ; \cdots ; L_{l} \leftarrow L_{l+1}, \ldots, L_{m}, \text { not } L_{m+1}, \ldots, \text { not } L_{n}
$$

( $n \geq m \geq l \geq 0$ ) where each $L_{i}$ is a positive/negative literal, i.e., $A$ or $\neg A$ for an atom $A$. not represents default negation or negation as failure (NAF). not $L$ is called an NAFliteral. Literals and NAF-literals are called LP-literals. The symbol ";" represents disjunction and "," represents conjunction. The rule (13) is read "if all $L_{l+1}, \ldots, L_{m}$ are believed and all $L_{m+1}, \ldots, L_{n}$ are disbelieved, then some of $L_{1}, \ldots, L_{l}$ is believed". The left-hand side of " $\leftarrow$ " is the head, and the right-hand side is the body. For each rule $r$ of the form (13), head(r), $\operatorname{body}^{+}(r)$ and $\operatorname{body}^{-}(r)$ denote the sets of literals $\left\{L_{1}, \ldots, L_{l}\right\}$, $\left\{L_{l+1}, \ldots, L_{m}\right\}$, and $\left\{L_{m+1}, \ldots, L_{n}\right\}$, respectively. Also, not_body ${ }^{-}(r)$ denotes the set of NAF-literals $\left\{\right.$ not $L_{m+1}, \ldots$, not $\left.L_{n}\right\}$. A disjunction of literals and a conjunction of (NAF-) literals in a rule are identified with its corresponding sets of literals. ${ }^{9}$ A rule $r$ is often written as

$$
\operatorname{head}(r) \leftarrow \operatorname{body}(r), \operatorname{not}_{-} \operatorname{body} \mathbf{y}^{-}(r) \quad \text { or } \quad h e a d(r) \leftarrow \operatorname{body}(r)
$$

where $\operatorname{body}(r)=\operatorname{body}^{+}(r) \cup n o t \_b o d y^{-}(r)$. A rule $r$ is disjunctive if head( $\left.r\right)$ contains more than one literal. A rule $r$ is a constraint if head $(r)=\emptyset$; and $r$ is a fact if $\operatorname{body}(r)=\emptyset$. A program is $N A F$-free if no rule contains NAF-literals. A rule $r_{1}$ subsumes a rule $r_{2}$ if head $\left(r_{1}\right) \theta \subseteq \operatorname{head}\left(r_{2}\right)$ and $\operatorname{body}\left(r_{1}\right) \theta \subseteq \operatorname{body}\left(r_{2}\right)$ hold for some substitution $\theta$. A program, rule, or literal is ground if it contains no variable. A program $P$ with variables is a shorthand of its ground instantiation Ground $(P)$, the set of ground rules obtained from $P$ by substituting variables in $P$ by elements of its Herbrand universe in every possible way. Two literals $L_{1}$ and $L_{2}$ have the same sign if both $L_{1}$ and $L_{2}$ are positive literals (or negative literals). A set $S$ of ground literals is consistent if $L \in S$ implies $\neg L \notin S$ for any literal $L$; otherwise, $S$ is contradictory. A set $S$ of literals satisfies a program $P$ if $b o d y^{+}(r) \subseteq S$ and $\operatorname{body}^{-}(r) \cap S=\emptyset$ imply head $(r) \cap S \neq \emptyset$ for any rule $r$ in $\operatorname{Ground}(P)$.

The semantics of an EDP is defined by the answer set semantics (Gelfond and Lifschitz 1991). Let Lit be the set of all ground literals in the language of a program. Suppose a program $P$ and a set of literals $S\left(\subseteq\right.$ Lit). Then, the reduct $P^{S}$ is the program which contains the ground rule head $(r) \leftarrow \operatorname{body}^{+}(r)$ iff there is a rule $r$ in Ground $(P)$ such that $b o d y^{-}(r) \cap$ $S=\emptyset$. Given an NAF-free EDP $P$, let $S$ be a set of ground literals that is (i) closed under $P$, i.e., for every ground rule $r$ in $\operatorname{Ground}(P), \operatorname{body}(r) \subseteq S$ implies $h e a d(r) \cap S \neq \emptyset$; and

\footnotetext{
${ }^{9} \mathrm{By}$ this fact, any duplicated appearance of the same literal in a rule is ignored. That is, a disjunction $(L ; L)$ is identified with $L$, a conjunction $(L, L)$ or $($ not $L$, not $L)$ is identified with $L$ or not $L$, respectively.
} 
(ii) logically closed, i.e., it is either consistent or equal to Lit. An answer set of an NAFfree EDP $P$ is a minimal set $S$ satisfying both (i) and (ii). Given an EDP $P$ and a set $S$ of ground literals, $S$ is an answer set of $P$ if $S$ is an answer set of $P^{S}$. A program has none, one, or multiple answer sets in general. The set of all answer sets of $P$ is written as $A S(P)$. An answer set is consistent if it is not Lit. A program $P$ is consistent if it has a consistent answer set; otherwise, $P$ is inconsistent.

Example 3.1 The program

$$
\begin{aligned}
& \text { tea } ; \text { coffee } \leftarrow, \\
& \text { milk } \leftarrow \text { tea, not lemon, } \\
& \text { lemon } \leftarrow \text { tea, not milk, } \\
& \text { milk } \leftarrow \text { coffee }
\end{aligned}
$$

has the three answer sets:

$$
\{\text { tea, milk\}, } \quad\{\text { tea, lemon }\}, \quad\{\text { coffee, } \text { milk\}, }
$$

which represent possible options for drink.

\subsection{Brave induction in ASP}

In this section, we consider the following problem setting:

- background knowledge $B$ is given as an EDP,

- an observation $O$ is given as a set of ground literals,

- a hypothesis $H$ is a finite set of rules.

Then, brave induction in ASP is defined as follows.

Definition 3.1 (Brave and cautious induction in ASP) Let $B$ be background knowledge and $O$ an observation. A hypothesis $H$ covers $O$ under $B$ in brave induction if $B \cup H$ has a consistent answer set $S$ such that $O \subseteq S$. $H$ is called a solution of brave induction. By contrast, $H$ covers $O$ under $B$ in cautious induction if $B \cup H$ is consistent and $O \subseteq S$ for any consistent answer set $S$ of $B \cup H$. $H$ is called a solution of cautious induction. ${ }^{10}$

Proposition 3.1 (Sufficient condition for the existence of solutions) Brave induction has a solution if $B \cup O$ is consistent. This is also the case for cautious induction.

Proof If $B \cup O$ is consistent, $B \cup O$ has a consistent answer set $S$. Then, $S$ becomes a consistent answer set of $B^{S} \cup O$ and $O \subseteq S$. By putting $H=O$, any consistent answer set $S$ of $B \cup H$ satisfies $O \subseteq S$.

In contrast to Proposition 2.2, the condition of Proposition 3.1 is not a necessary one.

\footnotetext{
${ }^{10}$ In nonmonotonic logic programming, logical connectives of classical logic are not used. So we write $B \cup H$ instead of $B \wedge H$.
} 
Example 3.2 Let $B=\{p \leftarrow q$, not $p\}$ and $O=\{q\}$. Then, $B \cup O$ has no answer set thereby inconsistent. But $H=\{q \leftarrow p, p \leftarrow\}$ becomes a solution of brave and cautious induction, since $B \cup H$ has the answer set $\{p, q\}$ in which $q$ is included.

Proposition 3.2 (Necessary condition for the existence of solutions) Brave induction has a solution only if $A S(B \cup O) \neq\{$ Lit $\}$.

Proof When $A S(B \cup O)=\{$ Lit $\}$, two cases are considered. (i) When $A S(B)=\{$ Lit $\}, B^{L i t}$ has the answer set Lit. This means that the set of NAF-free rules in $B$ is contradictory, so that there is no way to recover consistency by introducing any $H$ to $B$. In this case, brave induction has no solution. (ii) Else if $A S(B) \neq\{$ Lit $\}$ but $A S(B \cup O)=\{$ Lit $\}$, the set of NAFfree rules in $B$ is consistent but $B^{L i t} \cup O$ is contradictory. This means that there is a literal $L$ such that $L \in O$ and $\neg L \in S$ for any minimal closed set $S$ of $B^{L i t} \cup O$. Suppose that there is a solution $H$ such that $B \cup H$ has a consistent answer set $T$ satisfying $O \subseteq T$. In this case, $T$ is a minimal closed set satisfying $B^{L i t}$, so $T$ is an answer set of $B^{L i t} \cup T$. Since $\neg L \in S$ for any minimal closed set $S$ of $B^{L i t} \cup O$ and $O \subseteq T, \neg L \in U$ for any minimal closed set $U$ of $B^{L i t} \cup T$. Here, $T \subseteq U$ holds. As $T$ is an answer set of $B^{L i t} \cup T, T$ is a minimal closed set of $B^{L i t} \cup T$ and $\neg L \in T$. By $L \in O \subseteq T, L \in T$ so $T$ is contradictory. This contradicts the assumption that $T$ is a consistent answer set. Thus, $B \cup H$ cannot have a consistent answer set $T$ satisfying $O \subseteq T$. Hence, brave induction has no solution. By (i) and (ii), if brave induction has a solution, $A S(B \cup O) \neq\{$ Lit $\}$.

By the proof of Proposition 3.2, it is observed that the same necessary condition holds for cautious induction.

Proposition 3.3 (Necessary condition of solutions) If $H$ is a solution of brave induction, $B \cup H \cup O$ is consistent. This is also the case for cautious induction.

Proof If $H$ is a solution of brave induction, $B \cup H$ has a consistent answer set $S$ satisfying $O \subseteq S$. In this case, $B^{S} \cup H^{S}$ has a consistent answer set $S$ satisfying $O \subseteq S$. Then, $S$ becomes an answer set of $B^{S} \cup H^{S} \cup O$, so $S$ is an answer set of $B \cup H \cup O$. Hence, $B \cup H \cup O$ is consistent. The case for cautious induction is proved in a similar way.

Proposition 3.4 (Relation between brave and cautious induction)

1. Brave induction has a solution iff cautious induction has a solution.

2. If $H$ is a solution of cautious induction, $H$ is a solution of brave induction. The converse implication also holds when $B \cup H$ contains neither disjunction nor NAF.

Proof If brave induction has a solution $H, B \cup H \cup O$ is consistent (Proposition 3.3). Put $H^{\prime}=H \cup O$. Then, every consistent answer set $S$ of $B \cup H^{\prime}$ satisfies $O \subseteq S$. Hence, cautious induction has a solution. Conversely, if cautious induction has a solution $H$, it is also a solution of brave induction. When $B \cup H$ contains neither disjunction nor NAF, $B \cup H$ has at most one answer set. In this case, brave induction coincides with cautious induction.

Some properties of brave or cautious induction follows. ${ }^{11}$

\footnotetext{
${ }^{11}$ We do not address the property of disjunction of solutions, since it requires a definition of disjunction of ASP programs.
} 
Proposition 3.5 (Conjunction of solutions) The fact that both $H_{1}$ and $H_{2}$ are solutions of brave induction does not imply that $\mathrm{H}_{1} \cup \mathrm{H}_{2}$ is a solution of brave induction. This is also the case for cautious induction.

Proposition 3.6 (Conjunctions of observations) The fact that $\mathrm{H}$ covers both $\mathrm{O}_{1}$ and $\mathrm{O}_{2}$ under $B$ implies that $H$ covers $O_{1} \cup O_{2}$ under $B$ in cautious induction. But this is not the case for brave induction.

Proof If $O_{1} \subseteq S$ and $O_{2} \subseteq S$ hold for any consistent answer set $S$ of $B \cup H$, then $O_{1} \cup O_{2} \subseteq$ $S$. A counter-example for the case of brave induction is Example 2.3.

There are algorithms for computing cautious induction in ASP (Sakama 2005). In what follows, we develop a procedure for computing brave induction in ASP. As the case of clausal theories, the problem of our interest is the case when $B$ has no answer set including $O$. It is worth noting that in case of clausal theories, the consistency of $B \wedge H$ implies the consistency of $B, H$, and $O$. On the other hand, in case of ASP, the consistency of $B \cup H$ implies the consistency of $O$, but it does not necessarily imply the consistency of $B$ or $H$. In fact, an inconsistent program $B$ having no answer set can recover consistency by introducing an appropriate $H$.

Example 3.3 Let $B=\{p \leftarrow$ not $p\}$. Then, $H=\{p\}$ recovers the consistency in $B \cup H$.

For a technical reason, however, we assume the consistency of $B$ in the rest of this section. In case of brave induction from clausal theories, inverse entailment is used for computing hypotheses. However, it is known that inverse entailment in classical logic is not applied to nonmonotonic logic programs (Sakama 2000). We then consider another method for computing possible hypotheses.

\section{Step 1: Computing ground hypotheses}

We first introduce a notion used in this step.

Definition 3.2 (Relevant) Let $L_{0}$ be a ground literal and $S$ a set of ground literals. Then, $L_{1} \in S$ is relevant to $L_{0}$ if either (i) const $\left(L_{0}\right) \cap \operatorname{const}\left(L_{1}\right) \neq \emptyset$, or (ii) for some literal $L_{2} \in S$, const $\left(L_{1}\right) \cap \operatorname{const}\left(L_{2}\right) \neq \emptyset$ and $L_{2}$ is relevant to $L_{0}$. Otherwise, $L_{1} \in S$ is irrelevant to $L_{0}$.

Given an observation $O$, let $\Theta=\{L \mid L \in$ Lit and $\operatorname{pred}(L)$ appears in $O\}$. Suppose that background knowledge $B$ has a consistent answer set $S$. Then, construct a finite and consistent set $R$ of ground rules satisfying the following conditions. For any rule $r \in R$,

1. head $(r) \subseteq O$ and for any $L \in O$, there is a rule $r \in R$ such that head $(r)=\{L\}$.

2. $\operatorname{body}^{+}(r)=\{L \mid L \in S$ and $L$ is relevant to the literal in head $(r)\}$.

3. $\operatorname{body}^{-}(r)=\{L \mid L \in L i t \backslash(S \cup \Theta)$ and $L$ is relevant to the literal in head(r) and appears in Ground $(P)\}$.

The third condition requires that no rule contains default negation of literals in $S \cup \Theta$. The reason is that if $b o d y^{-}(r)$ contains literals from $S, \operatorname{body}(r)$ may contain both $L$ in $b o d y^{+}(r)$ and not $L$ in $b o d y^{-}(r)$, which makes the rule meaningless. Also, if $b o d y^{-}(r)$ contains literals from $\Theta, r$ may contain a negative loop that would make a program inconsistent. By its construction, different hypotheses are constructed by different answer sets in general. 


\section{Step 2: Generalization}

The notion of LGS is extended to rules containing default negation. It is done by syntactically viewing rules as "clauses". That is, identify disjunction ";" with the classical one " $\vee$, and any NAF-literal "not $p\left(t_{1}, \ldots, t_{n}\right)$ " with a new atom "not_p $\left(t_{1}, \ldots, t_{n}\right)$ " with the predicate "not_p". $\neg p$ is also considered a predicate " $\neg \_p$ " and is considered a predicate different from $p$. With this setting, the notion of comparable set of rules is defined as Definition 2.3, and the LGS of a comparable set of rules is defined in the same manner as the one in clausal theories (Sakama 2001). The generalization phase is similar to the case of clausal theories. For the set $R$ of rules obtained by the Step 1, $R$ is partitioned as $R=R_{1} \cup \cdots \cup R_{n}$ where each $R_{i}(1 \leq i \leq n)$ is a comparable set of ground rules. Then, the LGS of each $R_{i}$ is computed and collected as

$$
\lg s(R)=\left\{\lg s\left(R_{1}\right), \ldots, \lg s\left(R_{n}\right)\right\}
$$

\section{Step 3: Constructing a weak form of hypotheses}

To construct a weak form of hypotheses, we introduce the notion of cardinality constraint rules (Niemelä et al. 1999). A cardinality constraint rule is a rule of the form:

$$
h\left\{L_{1}, \ldots, L_{l}\right\} k \leftarrow L_{l+1}, \ldots, L_{m}, \text { not } L_{m+1}, \ldots, \text { not } L_{n}
$$

where $h$ and $k$ are two integers such that $h \leq k$. The rule (14) means if the body holds then at least $h$ and at most $k$ literals in the head hold. This type of rules is useful for representing knowledge in ASP and is used in the smodel system (Niemelä et al. 1999). A program with this type of rules is translated into a semantically equivalent EDP.

Given $\lg s\left(R_{1}\right), \ldots, \lg s\left(R_{n}\right)$, we construct a cardinality constraint rule

$$
1 \Sigma k \leftarrow \Gamma
$$

where $\Sigma=\operatorname{head}\left(\operatorname{lgs}\left(R_{1}\right)\right) \cup \cdots \cup \operatorname{head}\left(\operatorname{lgs}\left(R_{n}\right)\right), \Gamma=\operatorname{body}\left(\lg s\left(R_{1}\right)\right) \cup \cdots \cup \operatorname{body}\left(\lg s\left(R_{n}\right)\right)$, and $k$ is the number of literals in $\Sigma$, i.e., $k=|\Sigma|$. We write $C C R\left(\operatorname{lgs}\left(R_{1}\right), \ldots, \lg s\left(R_{n}\right)\right)$ to represent a cardinality constraint rule (15) constructed by $\lg s\left(R_{1}\right), \ldots, \lg s\left(R_{n}\right)$.

\section{Step 4: Optimization}

Optimization is done in two steps. First, remove any isolated literal from the body of any rule $\lg s\left(R_{i}\right)(1 \leq i \leq n)$. Here, the notion of "isolated literal" in a rule is defined by replacing a clause with a rule, and an atom with a literal in Definition 2.4. Second, remove any rule $\lg s\left(R_{i}\right)(1 \leq i \leq n)$ that is subsumed by another rule $\lg s\left(R_{j}\right)(1 \leq i \leq n)$. Let $\operatorname{lgs}^{*}\left(R_{i}\right)$ be the result of such reduction over $\lg s\left(R_{i}\right)$. When $B \cup\left\{\lg s\left(R_{i}\right)\right\}$ is consistent, the reduction is performed as far as $B \cup\left\{l g s^{*}\left(R_{i}\right)\right\}$ is consistent. Finally, put

$$
H^{\wedge}=\bigcup_{i}\left\{\lg s^{*}\left(R_{i}\right)\right\}
$$

if $B \cup \bigcup_{i}\left\{l g s^{*}\left(R_{i}\right)\right\}$ is consistent. Also, put

$$
H^{\vee}=\left\{C C R\left(\lg s^{*}\left(R_{1}\right), \ldots, \lg s^{*}\left(R_{n}\right)\right)\right\}
$$

if $B \cup\left\{C C R\left(\operatorname{lgs} s^{*}\left(R_{1}\right), \ldots, \operatorname{lgs}^{*}\left(R_{n}\right)\right)\right\}$ is consistent.

The algorithm of brave induction in ASP (called BRAIN ${ }^{n o t}$ ) is sketched in Fig. 2. In what follows, we show that BRAIN ${ }^{\text {not }}$ computes hypotheses for brave induction in ASP. We say that $O$ is independent of $B$ if every predicate in $O$ appears nowhere in $B$. 


\author{
Procedure: BRAIN ${ }^{\text {not }}$ \\ Input : the background knowledge $B$ and an observation $O$; \\ Output : hypotheses $H^{\wedge}$ and $H^{\vee}$. \\ Step 1 : Select an answer set $S$ of $B$ and construct a set $R$ of rules. \\ Step 2 : Compute $\operatorname{lgs}(R)$. \\ Step 3 : Compute $C C R\left(\operatorname{lgs}\left(R_{1}\right), \ldots, \lg s\left(R_{n}\right)\right)$. \\ Step 4 : Produce $l g s^{*}\left(R_{i}\right)$ by reduction, and compute $H^{\wedge}$ and $H^{\vee}$.
}

Fig. 2 An algorithm for brave induction in ASP

Lemma 3.7 Let $B$ be background knowledge and $O$ an observation. If $O$ is independent of $B, B \cup H^{\wedge}$ has an answer set $U$ such that $O \subseteq U$.

Proof Let $S$ be a consistent answer set of $B$. For any rule $r$ in $R, \operatorname{head}(r) \leftarrow \operatorname{body}^{+}(r)$ is in $R^{S}$. Here, $\operatorname{body}^{+}(r) \subseteq S$, head $(r) \subseteq O$, and for any $L \in O$, $\operatorname{pred}(L)$ appears nowhere in $B$. Put $T=S \cup\left\{L \mid L \in\right.$ head $(r)$ and $\left.r \in R^{S}\right\}$. By $B^{T} \cup R^{T}=B^{S} \cup R^{T}, T$ becomes a minimal closed set of $B^{T} \cup R^{T}=(B \cup R)^{T}$. Since $O$ is independent of $B$, every predicate in head $(r)$ appears nowhere in $B$ so that $T$ is a consistent set of literals. As every literal in $O$ appears in the head of some rule $r$ in $R, T$ is a consistent answer set of $B \cup R$ such that $O \subseteq T$. Next, we show that $B \cup \operatorname{lgs}(R)$ has a consistent answer set such that $O \subseteq U$. Let $R=R_{1} \cup \cdots \cup R_{n}$. By the definition, $\lg s\left(R_{i}\right) \theta \subseteq r$ for any $r \in R_{i}(1 \leq i \leq n)$ with some ground substitution $\theta$. Then, for any rule $r \in R_{i}, \operatorname{body}^{-}(r) \cap S=\emptyset$ implies body ${ }^{-}\left(\lg s\left(R_{i}\right) \theta\right) \cap S=\emptyset$. So $B^{S} \cup R^{S} \subseteq$ $B^{S} \cup \lg s(R)^{S}$. Since $O$ is independent of $B, \lg s(R)^{S} \backslash R^{S}$ is a set of NAF-free rules whose heads have predicates appearing nowhere in $B$. Put $V=\left\{L \mid r \in \lg s(R)^{S} \backslash R^{S}\right.$, head $(r)=$ $\{L\}$ and $\left.b_{0 d y}(r) \subseteq S\right\}$. Then, $B^{S \cup V} \cup \operatorname{lgs}(R)^{S \cup V}=B^{S} \cup \lg s(R)^{S}$ has a minimal closed set $U=S \cup V$. Since $O$ is independent of $B, S \cup V$ is consistent. As any literal in $O$ appears in the head of some ground instance of a rule in $B \cup \operatorname{lgs}(R), U$ is a consistent answer set of $B \cup \operatorname{lgs}(R)$ such that $O \subseteq U$. When $B \cup \bigcup_{i}\left\{l g s^{*}\left(R_{i}\right)\right\}$ is consistent, $U$ also becomes a consistent answer set of $B \cup H^{\wedge}$. Hence, the result follows.

Lemma 3.8 Let $B$ be background knowledge and $O$ an observation. If $O$ is independent of $B, B \cup H^{\vee}$ has an answer set $U$ such that $O \subseteq U$.

Proof Let $r=C C R\left(\lg s\left(R_{1}\right), \ldots, \lg s\left(R_{n}\right)\right)$. For any ground instance of $r \theta$ satisfying body ${ }^{+}(r \theta) \subseteq S$ and $b o d y^{-}(r \theta) \cap S=\emptyset$ for some answer set $S$ of $B$, a set $T$ of literals is constructed in a way that a literal $L$ is selected from the head of each $r \theta$ whenever $L \in O$. Since $O$ is independent of $B, S \cup T$ is consistent. Then, $U=S \cup T$ becomes a consistent answer set of $B \cup\left\{C C R\left(\lg s\left(R_{1}\right), \ldots, \lg s\left(R_{n}\right)\right)\right\}$ and $O \subseteq U$. When $B \cup\left\{C C R\left(\lg s^{*}\left(R_{1}\right), \ldots, \lg s^{*}\left(R_{n}\right)\right)\right\}$ is consistent, $U$ also becomes a consistent answer set of $B \cup H^{\vee}$.

By Lemmas 3.7 and 3.8, we have the next result.

Theorem 3.9 (Soundness) Any hypothesis computed by BRAIN ${ }^{\text {not }}$ becomes a solution of brave induction.

BRAIN $^{\text {not }}$ is incomplete with respect to solutions of brave induction, since it reduces seemingly useless hypotheses in the optimization phase. 
Example 3.4 There are two couples, Adam and Nancy, and Bob and Jane. They plan to go to either sea or mountain on this weekend. Each couple can select one of them, but a husband and a wife go to the same place. The situation is represented as background knowledge $B$ :

$$
\begin{aligned}
& s(x) \leftarrow \operatorname{not} m(x), \\
& m(x) \leftarrow \operatorname{not} s(x), \\
& c(a, n) \leftarrow, \\
& c(b, j) \leftarrow, \\
& \leftarrow c(x, y), s(x), m(y), \\
& \leftarrow c(x, y), s(y), m(x)
\end{aligned}
$$

where the predicates $s, m$ and $c$ mean sea, mountain and couple, respectively, and the constants $a, n, b$ and $j$ mean Adam, Nancy, Bob and Jane, respectively. $B$ has four answer sets:

$$
\begin{aligned}
& S_{1}=\{c(a, n), c(b, j), s(a), s(n), s(b), s(j)\}, \\
& S_{2}=\{c(a, n), c(b, j), s(a), s(n), m(b), m(j)\}, \\
& S_{3}=\{c(a, n), c(b, j), m(a), m(n), s(b), s(j)\}, \\
& S_{4}=\{c(a, n), c(b, j), m(a), m(n), m(b), m(j)\} .
\end{aligned}
$$

Suppose the observation that Adam and Nancy are tanned, but Bob and Jane are not. It is represented as:

$$
O=\{t(a), t(n), \neg t(b), \neg t(j)\}
$$

where the predicate $t$ mean tanned.

BRAIN $^{\text {not }}$ constructs candidate hypotheses as follows.

(Step 1) First, an answer set of $B$, for instance $S_{2}$, is selected. A set $R$ of rules is then constructed as:

$$
\begin{aligned}
& t(a) \leftarrow c(a, n), s(a), s(n), \operatorname{not} m(a), \operatorname{not} m(n), \\
& t(n) \leftarrow c(a, n), s(a), s(n), \operatorname{not} m(a), \operatorname{not} m(n), \\
& \neg t(b) \leftarrow c(b, j), m(b), m(j), \operatorname{not} s(b), \operatorname{not} s(j), \\
& \neg t(j) \leftarrow c(b, j), m(b), m(j), \operatorname{not} s(b), \operatorname{not} s(j) .
\end{aligned}
$$

Note that the body of each rule contains literals that are relevant to the literal in the head.

(Step 2) Next, the $\lg s(R)$ is constructed as

$$
\begin{aligned}
& t(x) \leftarrow c(a, n), s(x), \operatorname{not} m(x), \\
& \neg t(y) \leftarrow c(b, j), m(y), \operatorname{not} s(y) .
\end{aligned}
$$

(Step 3) Third, from the rules in $\lg s(R)$, the cardinality constraint rule is constructed as

$$
1\{t(x), \neg t(y)\} 2 \leftarrow c(a, n), c(b, j), s(x), m(y), \operatorname{not} m(x), \operatorname{not} s(y) .
$$


(Step 4) Finally, isolated literals $c(a, n)$ and $c(b, j)$ are removed, and $H^{\wedge}$ and $H^{\vee}$ become

$$
\begin{aligned}
& H^{\wedge}=\{t(x) \leftarrow s(x), \operatorname{not} m(x), \neg t(y) \leftarrow m(y), \operatorname{not} s(y)\}, \\
& H^{\vee}=\{1\{t(x), \neg t(y)\} 2 \leftarrow s(x), m(y), \operatorname{not} m(x), \operatorname{not} s(y)\},
\end{aligned}
$$

which are two solutions of brave induction.

\section{Computational complexity}

In this section, we consider computational complexity of brave induction. Throughout the section, we assume that background knowledge, hypotheses, and observations are all represented over a finite propositional language. ${ }^{12}$

The following two decision problems are considered.

- Existence: Given background knowledge $B$ and an observation $O$, deciding whether $O$ has a solution of brave induction under $B$.

- Verification: Given background knowledge $B$ and an observation $O$, deciding whether a given hypothesis $H$ is a solution of brave induction under $B$.

We consider these problems in the context of clausal theories (CT) and answer set programming (ASP), and compare complexity results between brave induction and cautious induction.

Theorem 4.1 In clausal theories the following complexity results hold.

1. Deciding the existence of solutions in brave induction is NP-complete. The same complexity result holds for cautious induction.

2. Deciding whether a given hypothesis is a solution of brave induction is $\Sigma_{2}^{P}$-complete, while the corresponding problem in cautious induction is coNP-complete.

Proof (1) By Proposition 2.2, brave induction has a solution $H$ iff $B \wedge O$ is consistent. The task of deciding the consistency of $B \wedge O$ is NP-complete. Since the existence of solutions coincides in brave and cautious induction, the same complexity result holds for cautious induction.

(2) To show the result, we consider a complementary problem: a ground clausal theory $B \wedge H$ has no minimal model satisfying a conjunction $O$ of ground atoms. This is a task of the extended GCWA and is known $\Pi_{2}^{P}$-complete (Eiter and Gottlob 1995), so that the verification problem is $\Sigma_{2}^{P}$-complete. In case of cautious induction, $H$ becomes a solution iff $B \wedge H \models O$ iff $B \wedge H \wedge \neg O$ is inconsistent. Deciding the unsatisfiability of $B \wedge H \wedge \neg O$ is coNP-complete.

To show complexity results in ASP, we introduce a program transformation.

Let $r$ be a ground rule of the form

$$
L_{1} ; \cdots ; L_{l} \leftarrow L_{l+1}, \ldots, L_{m}, \text { not } L_{m+1}, \ldots, \text { not } L_{n}
$$

\footnotetext{
${ }^{12}$ We view a program with variables as a shorthand of its ground instantiation. When the language contains no function symbol, the ground instantiation of a program is finite. In this case, the ground instantiated program is identified with a finite propositional program.
} 
( $n \geq m \geq l \geq 0$ ). The propositional formula $\phi(r)$ associated with $r$ is defined as

$$
L_{l+1}^{\prime} \wedge \cdots \wedge L_{m}^{\prime} \wedge \neg L_{m+1}^{\prime} \wedge \cdots \wedge \neg L_{n}^{\prime} \supset L_{1}^{\prime} \vee \cdots \vee L_{l}^{\prime}
$$

where $L_{i}^{\prime}=L_{i}$ if $L_{i}$ is a positive literal, and $L_{j}^{\prime}=\bar{A}$ if $L_{j}$ is a negative literal $\neg A(1 \leq i$, $j \leq n)$. Thus, any negative literal $\neg A$ in $r$ is transformed to a new atom $\bar{A}$, and default negation not $L$ is transformed to a negative literal $\neg L$ in $\phi(r)$. Given a ground EDP $P$, we define a propositional theory $\phi(P)$ as

1. for any $r \in P, \phi(r)$ is in $\phi(P)$.

2. for any positive literal $L \in L i t$, the following formula is in $\phi(P)$

$$
L \wedge \bar{L} \supset \text { false. }
$$

Given a set $S$ of literals, $\bar{S}$ is the set of atoms which is obtained from $S$ by replacing every negative literal $\neg L$ with the corresponding atom $\bar{L}$. Conversely, given a set $M$ of atoms, $M^{\urcorner}$is the set of literals which is obtained from $M$ by replacing every atom $\bar{L}$ with the corresponding negative literal $\neg L$.

Proposition 4.2 If an EDP $P$ is consistent, so is $\phi(P)$.

Proof If $P$ is consistent, there is a consistent minimal set $S$ of literals such that $\left\{L_{l+1}, \ldots\right.$, $\left.L_{m}\right\} \subseteq S$ and $\left\{L_{m+1}, \ldots, L_{n}\right\} \cap S=\emptyset$ imply $\left\{L_{1}, \ldots, L_{l}\right\} \cap S \neq \emptyset$ for any rule (16) in $P$. In this case, $\bar{S}$ satisfies both (17) and (18). Hence, $\phi(P)$ is consistent.

Theorem 4.3 In ASP the following complexity results hold.

1. Deciding the existence of solutions in brave induction is NP-complete. The same complexity result holds for cautious induction.

2. Deciding whether a given hypothesis is a solution of brave induction is $\Sigma_{2}^{P}$-complete, while the corresponding problem in cautious induction is $\Pi_{2}^{P}$-complete.

Proof (1) Given an EDP $B$ and a set $O$ of ground literals, we show that brave induction has a solution iff the propositional theory $\phi(B \cup O)$ is consistent. Suppose that $\phi(B \cup O)$ is consistent. Then, $\phi(B \cup O)$ has a model $M$ satisfying the following three conditions: (i) $\bar{O} \subseteq M$, (ii) for any formula (17) in $\phi(B),\left\{L_{l+1}^{\prime}, \ldots, L_{m}^{\prime}\right\} \subseteq M$ and $\left\{L_{m+1}^{\prime}, \ldots, L_{n}^{\prime}\right\} \cap$ $M=\emptyset$ imply $\left\{L_{1}^{\prime}, \ldots, L_{l}^{\prime}\right\} \cap M \neq \emptyset$, and (iii) for any atom $L,\{L, \bar{L}\} \nsubseteq M$ by (18). In this case, $M^{\urcorner}$is a consistent set of literals satisfying $B \cup O$. Then, $M^{\urcorner}$is a consistent minimal closed set satisfying $B^{M\urcorner} \cup M^{\urcorner}$. Thus, $M^{\urcorner}$is an answer set of $B^{M\urcorner} \cup M^{\urcorner}$and $O \subseteq M\urcorner$. Putting $H=M^{\urcorner}, H$ is a solution of brave induction. Conversely, suppose that brave induction has a solution. Then, for any solution $H$ of brave induction, $B \cup H \cup O$ is consistent (Proposition 3.3). In this case, $\phi(B \cup H \cup O)$ ) is consistent (Proposition 4.2), so that $\phi(B \cup O)$ is consistent. Hence, brave induction has a solution iff $\phi(B \cup O)$ is consistent. Since deciding the consistency of the propositional theory $\phi(B \cup O)$ is NP-complete, the result holds. By Proposition 3.4, brave induction has a solution iff cautious induction has a solution. Hence, the same complexity result holds for cautious induction.

(2) Deciding whether some (resp. every) consistent answer set $S$ of $B \cup H$ satisfies $O \subseteq S$ is $\Sigma_{2}^{P}$-complete (resp. $\Pi_{2}^{P}$-complete) (Eiter and Gottlob 1995). Hence, the result holds.

The complexity results are summarized in Table 2 . In the table, every entry represents completeness for the respective class. These complexity results show that brave and cautious 
Table 2 Computational complexity

\begin{tabular}{lllll}
\hline & \multicolumn{2}{c}{ Brave induction } & \multicolumn{2}{c}{ Cautious induction } \\
Language & $\mathrm{CT}$ & $\mathrm{ASP}$ & $\mathrm{CT}$ & ASP \\
\hline Existence & $\mathrm{NP}$ & $\mathrm{NP}$ & $\mathrm{NP}$ & $\mathrm{NP}$ \\
Verification & $\Sigma_{2}^{P}$ & $\Sigma_{2}^{P}$ & $\mathrm{coNP}$ & $\Pi_{2}^{P}$ \\
\hline
\end{tabular}

induction are in the same complexity class for checking the existence of solutions. It is worth noting that extending the language from CT to ASP does not lead to a complexity increase in this problem. On the other hand, for the task of solution verification, the complexity of brave induction is one level higher in the polynomial hierarchy than that of cautious induction in CT. By contrast, the complexities of brave induction and cautious induction are at the same level of the polynomial hierarchy in ASP.

\section{Problem solving by brave induction}

\subsection{Systems biology}

In this section, we show the use of brave induction for inference of master reactions from biochemical networks in systems biology. It is a crucial feature of flux distributions that metabolic reactions with fluxes spanning several orders of magnitude coexist under the same conditions (Almaas et al. 2004). Although most metabolic reactions have low fluxes, the overall behavior of metabolism is dominated by several reactions with very high fluxes. In (Yamamoto et al. 2009), the states of each enzyme reaction is simply divided into two kinds, activated and non-activated, in order to analyze which chemical reactions have high fluxes. This analysis is helpful to solve the differential equations associated with reactions by ignoring non-activated reactions with low fluxes. We apply brave induction to hypothesis-finding in biochemical networks. Consider a simple chemical reaction represented in Fig. $3 .^{13}$

In Fig. 3, the two reactions involve the same substrate $s_{1}$ and are catalyzed by the enzymes $e_{1}$ and $e_{2}$, then lead to the products $p_{1}$ and $p_{2}$, respectively. These two reactions are represented by the formulas:

$$
R=\left\{\operatorname{reaction}\left(e_{1}, s_{1}, p_{1}\right), \text { reaction }\left(e_{2}, s_{1}, p_{2}\right)\right\} .
$$

Assume that the levels of concentration of compounds are classified into five, $l_{1}, \ldots, l_{5},{ }^{14}$ and that the concentration levels of the products $p_{1}$ and $p_{2}$ are as follows:

$$
O=\left\{\text { concentration }\left(p_{1}, l_{1}\right) \text {, concentration }\left(p_{2}, l_{2}\right)\right\} \text {. }
$$

Fig. 3 Two reactions with one substrate and one product

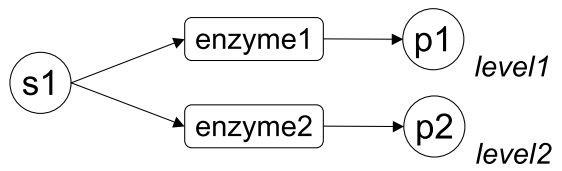

\footnotetext{
${ }^{13}$ This example is given by Yoshitaka Yamamoto.

${ }^{14}$ On logical representation of concentration change, we here subdivide the levels into five or more instead of qualitative two-valued expression like "up" and "down" in (Yamamoto et al. 2009). With this refinement, more precise representation becomes possible. This is advised by Andrei Doncescu.
} 
Next, suppose that the two enzymes $e_{1}$ and $e_{2}$ are of the same type $t$ :

$$
E=\left\{\operatorname{class}\left(e_{1}, t\right), \operatorname{class}\left(e_{2}, t\right)\right\} .
$$

Let background knowledge be $B=R \cup E$. Then, the next hypothesis $H$ becomes a solution of brave induction.

$$
\begin{aligned}
H= & \left\{\text { concentration }\left(Y, l_{1}\right) \vee \text { concentration }\left(Y, l_{2}\right)\right. \\
& \leftarrow \text { reaction }(\text { Enzyme }, X, Y) \wedge \operatorname{class}(\operatorname{Enzyme}, t)\} .
\end{aligned}
$$

The above $H$ cannot be induced by explanatory or cautious induction. The hypothesis $H$ represents that an enzyme reaction of the type $t$ leads to a product with low-level concentration that is either at level $l_{1}$ or $l_{2}$. In other words, an enzyme of the type $t$ is non-activated or is inhibited by some reason. Note that, if the concentration level of $p_{1}$ is the same as that of $p_{2}$, ordinary systems of explanatory induction can also induce an appropriate hypothesis. However, when we consider multiple levels of concentration, the hypothesis by brave induction is considered more useful.

In general, brave induction can induce a causal rule which combines multiple states as alternative effects. To infer master reactions correctly from biochemical pathways, it is necessary to set background knowledge appropriately. This task often involves abduction (Tamaddoni-Nezhad et al. 2006; Yamamoto et al. 2009), but causal rules given in background knowledge are often incomplete. Brave induction can thus be useful to complete missing causal rules in these applications.

\subsection{Requirements engineering}

Requirements engineering involves the elicitation of high-level stakeholder goals that are described by scenarios of desirable and undesirable system behavior. Alrajeh et al. (2007) introduce an ILP framework for inferring requirements from a set of scenarios and incomplete requirements specification. Scenarios represent examples of desirable and undesirable system behavior over time, while the requirements specification captures the initial but incomplete background knowledge of the envisioned system and its environment. The task is then to complete the specification by learning a set of missing requirements that cover all of the desirable scenarios but none of the undesired ones. Formally, the problem is specified as follows:

Given: a requirement specification $S p e c$, a set Des of desirable scenarios, and a set Und of undesirable scenarios

Find: a set Pre of event precondition axioms satisfying the conditions:

- Spec $\cup$ Pre $\models_{M} \neg P_{u}$ for any $P_{u} \in U n d$,

- Spec $\cup$ Pre $\not \nvdash_{M} \neg P_{d}$ for any $P_{d} \in$ Des,

where $\models_{M}$ means an entailment relation under an $\operatorname{LTL}^{15}$ model $M$.

Any set of event precondition axioms that satisfy these two properties is said to be a correct extension of a requirements specification with respect to the given scenarios. The specification and scenarios are represented by event calculus normal logic programs. They

${ }^{15}$ Linear Temporal Logic. 
compute Pre satisfying that Spec $\cup$ Pre has a stable model $M$ such that every element in Und is false $M$ and every element in Des is consistent with $M$. Incidentally, their program transformation produces a normal logic program Spec $\cup$ Pre which has a single stable model, but it is inherently a problem of brave induction.

\subsection{Multiagent negotiation}

Negotiation is a process of reaching agreement between different agents. In a typical oneto-one negotiation, an agent makes a proposal on his/her request and the opponent agent decides whether it is acceptable or not. If a proposal is unacceptable as it is, an agent seeks conditions to accept it by extending his/her current belief to accommodate another agent's request.

Sakama (2008) formulates the process of building conditions in terms of induction. Given the current belief $B$ of an agent and a proposal $G$ of another agent, $B$ could accept $G$ under the condition $H$ if:

$$
B \cup H \models G
$$

where $B \cup H$ is consistent. Here, $H$ is a condition that bridges the gap between the current belief of an agent and the request made by another agent. Viewing $G$ as an observation, the problem of finding $H$ is considered a process of building a hypothesis to explain $G$ under $B$.

When $B$ contains multiple minimal models or answer sets, however, the relation $B \cup H \models$ $G$ is strong. This is because an agent would have alternative options for a deal, and the cautious inference requires that the proposal $G$ must be satisfied in every possible option. To relax the condition, Sakama uses brave induction for negotiation. That is, $B$ could accept $G$ under the condition $H$ if $B \cup H$ has an answer set satisfying $G$.

Example 5.1 Consider negotiation between a buyer and a seller. A seller agent has the knowledge base $B$ which consists of the following rules:

$$
\begin{aligned}
& \operatorname{product}(p c, \$ 1500) \leftarrow \operatorname{not} \neg \operatorname{product}(p c, \$ 1500), \\
& \leftarrow \operatorname{product}(p c, x), \operatorname{product}(p c, y), x \neq y, \\
& \neg \operatorname{product}(p c, \$ 1500) \leftarrow \operatorname{product}(p c, x), x<\$ 1500, p a y \_c a s h, \\
& \text { pay_cash } ; \text { pay_card } \leftarrow .
\end{aligned}
$$

Here, the rule (19) represents that the normal price of a PC is 1500 USD. The rule (20) represents a constraint that the same pc cannot have different prices at the same time. The rule (21) represents if discount is made by payment with cash, the normal price is withdrawn. The rule (22) represents two options for payment. With this setting, $B$ has two answer sets:

$$
\begin{aligned}
& S_{1}=\{\operatorname{product}(p c, \$ 1500), \text { pay_cash }\}, \\
& S_{2}=\left\{\operatorname{product}(p c, \$ 1500), p a y \_c a r d\right\},
\end{aligned}
$$

which represent the seller's initial belief.

Next, suppose that a buyer proposes

$$
G: \operatorname{product}(p c, \$ 1300)
$$


to the seller. As $G$ is included in no answer set of $B$, the seller cannot accept $G$ as it is. The seller then seeks a condition $H$ to accept $G$ and induces the hypothesis

$$
H: \operatorname{product}(p c, \$ 1300) \leftarrow \text { pay_cash } .
$$

Now $B \cup H$ has two answer sets:

$$
\begin{aligned}
S_{3} & =\left\{\operatorname{product}(p c, \$ 1300), \neg \operatorname{product}(p c, \$ 1500), \operatorname{pay} \_c a s h\right\}, \\
S_{4} & =\left\{\operatorname{product}(p c, \$ 1500), p a y \_c a r d\right\}
\end{aligned}
$$

of which $S_{3}$ satisfies $G$. Thus, $H$ covers $G$ under $B$ in brave induction. Based on $H$, the seller returns the condition

$$
G^{\prime}: \text { pay_cash }
$$

as a counter-proposal.

\section{Discussion}

In the previous sections, we mainly compared brave induction with explanatory or cautious induction. Here, we compare brave induction with other forms of induction.

\subsection{Learning from satisfiability}

De Raedt and Dehaspe (De Raedt 1997; De Raedt and Dehaspe 1997b) introduce the framework of learning from satisfiability (LFS). Given background knowledge $B$ and an observation $O$, a hypothesis $H$ covers $O$ under $B$ in LFS iff $B \wedge H \wedge O$ is consistent. In other words, $H$ covers $O$ under $B$ in LFS iff $B \wedge H$ has a model satisfying $O$. As already argued in the introduction, LFS is weaker than brave induction.

Proposition 6.1 If a hypothesis $H$ covers $O$ under $B$ in brave induction, $H$ covers $O$ under $B$ in LFS.

Proof The result holds by Proposition 2.3.

The converse implication of Proposition 6.1 does not hold in general. Since brave induction is weaker than both explanatory and cautious induction (Proposition 2.1), the following relation holds.

$$
\begin{aligned}
& \text { explanatory induction }<\text { cautious induction } \\
& <\text { brave induction }<\text { learning from satisfiability }
\end{aligned}
$$

where $X<Y$ means that any solution of $X$ is also a solution of $Y$, but not vice versa.

Compared with brave induction, LFS does not require the minimality of models. So any theory $H$ becomes a solution as far as it is consistent with $B \wedge O$. Due to its weak condition, the hypothesis space for LFS is generally huge, and additional language bias would be necessary for practical usage. Brave induction is considered as a strengthened version of LFS, that is, we imposed the condition of minimality on models of $B \wedge H$ satisfying $O$. It is known that LFS does not satisfy the property of "conjunction of solutions" in Sect. 2.1 (De Raedt and Dehaspe 1997b). The following example illustrates that LFS also does not satisfy the property of "conjunction of observations". 
Example 6.1 Let $B=\{p(a)\}, O_{1}=\{q(a)\}$ and $O_{2}=\{r(a)\}$. Then, $H=\{q(a) \vee r(a) \leftarrow$ $p(a), \leftarrow q(a) \wedge r(a)\}$ covers both $O_{1}$ and $O_{2}$ under $B$ in LFS, but $H$ does not cover $\mathrm{O}_{1} \wedge \mathrm{O}_{2}$.

Thus, as for the properties of Table 1 in Sect. 2.1, LFS is the same as brave induction. In (De Raedt and Dehaspe 1997b) the authors say:

One open question for further research is how learning from satisfiability (which employs a monotonic logic) could be used for inducing nonmonotonic logic programs.

In Definition 3.1, brave induction is defined as inducing hypothesis $H$ such that $B \cup H$ has a consistent answer set $S$ satisfying $O \subseteq S$. The definition is considered a strengthened version of LFS in nonmonotonic logic programs.

\subsection{Learning from interpretations and confirmatory induction}

As argued in the introduction, learning from interpretations (LFI) realizes a different style of induction. When background knowledge $B$ is given as a definite clause theory, a hypothesis $H$ covers $O$ under $B$ in LFI iff $H$ is satisfied in the least model of $B \wedge O$ (De Raedt and Dehaspe 1997a). On the other hand, when $B$ is a full clausal theory, Helft (1989) distinguishes two types of induction. ${ }^{16}$ Strong generalization is a set $H$ of clauses which are satisfied in every minimal model of $B \wedge O$, while weak generalization is a set $H$ of clauses which are satisfied in some minimal model of $B \wedge O .{ }^{17}$

Example 6.2 Consider background knowledge $B$ and an observation $O$,

$$
\begin{aligned}
& B: \text { american }(J o h n) \vee \text { english }(J o h n), \\
& O: \text { speak_english }(J o h n) .
\end{aligned}
$$

Then,

$$
\begin{aligned}
& H_{1}: \operatorname{american}(x) \leftarrow \text { speak_english }(x), \\
& H_{2}: \text { english }(x) \leftarrow \text { speak_english }(x)
\end{aligned}
$$

are two weak generalizations, while

$$
H_{3}: \operatorname{american}(x) \vee \text { english }(x) \leftarrow \text { speak_english }(x)
$$

is a strong generalization.

In Example 6.2, none of $H_{1}, H_{2}$, and $H_{3}$ becomes a solution of brave induction. Solutions by brave induction are, for instance,

$$
\begin{aligned}
& H_{4}: \text { speak_english }(x) \leftarrow \operatorname{american}(x), \\
& H_{5}: \text { speak_english }(x) \leftarrow \text { english }(x),
\end{aligned}
$$

\footnotetext{
${ }^{16}$ Helft's semantics is often called nonmonotonic ILP, but we reserve the term for induction from nonmonotonic logic programs. Helft's semantics is similar to LFI in spirit (De Raedt and Dehaspe 1997a), while it is also viewed as an instance of confirmatory induction (De Raedt and Lavrač 1993; Lachiche 2000).

${ }^{17}$ Helft imposes additional conditions on the satisfiability of $H$ in a model of $B \wedge O$, but we neglect them to make discussion simple.
} 
which are also solutions of LFI. Note that $H_{4}$ or $H_{5}$ does not become a solution of cautious induction, while $\mathrm{H}_{4} \cup \mathrm{H}_{5}$ becomes a solution of cautious induction.

On the other hand, if the fact american (Mary) is added to $B, H_{4}$ and $H_{5}$ are still solutions of brave induction but $H_{4}$ is not a solution of LFI anymore. This is because $H_{4}$ is not satisfied in $B \cup\{$ american(Mary)\}. Thus, brave induction is neither stronger nor weaker than LFI. Generally speaking, LFI does not explain why particular individuals are observed under background knowledge. In fact, LFI does not distinguish between $B$ and $O$. Moreover, LFI assumes that all observations are completely specified, so that it has no mechanism of predicting unseen phenomena. This is in contrast to brave or cautious induction which has a mechanism of prediction.

Confirmatory induction or descriptive induction (Lachiche 2000) also builds hypotheses that are satisfied by observations. Given background knowledge $B$ and an observation $O$ such that $B \wedge O$ is consistent, a hypothesis $H$ covers $O$ under $B$ in confirmatory induction iff $\operatorname{Comp}(B \wedge O) \models H$ where Comp represents Clark's predicate completion (Clark 1978).

Example 6.3 Given $B$ and $O$ of Example 6.2, $\operatorname{Comp}(B \wedge O)$ becomes

$$
\begin{aligned}
& \operatorname{american}(x) \Leftrightarrow x=\operatorname{John} \wedge \neg \text { english(John), } \\
& \operatorname{english}(x) \Leftrightarrow x=\operatorname{John} \wedge \neg \operatorname{american}(\text { John }), \\
& \text { speak_english }(x) \Leftrightarrow x=J o h n,
\end{aligned}
$$

together with the Clark's equality axioms. Thus, $H_{1}, \ldots, H_{5}$ are all solutions of confirmatory induction.

Like LFI, brave induction is neither stronger nor weaker than confirmatory induction in general.

\subsection{Induction in nonmonotonic logic programs}

Otero (2001) introduces a framework for learning positive/negative examples in normal logic programs. ${ }^{18} \mathrm{He}$ considers induction from several sets of examples such that: given a normal logic program $P$ and several sets of examples $E_{1}, \ldots, E_{n}$ where $E_{i}=E_{i}^{+} \cup E_{i}^{-}(1 \leq$ $i \leq n), H$ is a solution of induction if there is a stable model $M_{i}$ of $P \cup H$ such that $M_{i} \models E_{i}^{+}$ and $M_{i} \not \models E_{i}^{-}$for each $E_{i}$.

Example 6.4 (Otero 2001) Consider the normal logic program $B$ :

$$
p \leftarrow \operatorname{not} q
$$

which has the unique stable model $\{p\}$. Given examples $E_{1}=\{p\}$ and $E_{2}=\{q\}, H=\{q \leftarrow$ not $p$ \} becomes a solution as $B \cup H$ has two stable models $E_{1}$ and $E_{2}$.

In Otero's setting, examples are given as multiple sets and each set of examples is examined to be satisfied by a stable model of a program. This is different from the problem setting of brave induction which examines satisfaction of a single set of examples in an answer set

\footnotetext{
${ }^{18}$ A normal logic program is a logic program in which a rule can contain default negation but contains neither negative literals nor disjunction.
} 
of a program. Otero's framework reduces to the definition of cautious induction for a single set of examples.

Induction in answer set programming is introduced by Sakama (2005), which builds new rules to cover positive examples and uncover negative examples. More precisely, given an extended logic program $B^{19}$ and a ground literal $L^{+}$(resp. $L^{-}$) as a positive (resp. negative) example, it finds a set $H$ of rules such that

$$
B \cup H \models L^{+} \quad \text { and } \quad B \cup H \not=L^{-}
$$

and $B \cup H$ is consistent. This definition provides a logical framework of cautious induction in ASP.

Ray (2008) develops a nonmonotonic ILP system, called XHAIL, which combines abduction and induction for building hypotheses. The background theory is given as a normal logic program, and its semantics is given by the stable model semantics. Given examples, XHAIL first computes explanations by brave abduction. Next, XHAIL constructs ground rules as hypotheses by putting abductive explanations in heads of rules and putting deductive consequences of $B$ in bodies of rules. In this phase, a mode declaration specifies atoms appearing in heads and bodies of possible hypotheses. Finally, the ground hypotheses are generalized in the inductive phase. The resulting hypothesis becomes a solution of brave induction because it is constructed from explanations of brave abduction. In this sense, it is said that XHAIL realizes brave induction. However, the paper (Ray 2008) does not mention any motivation of brave induction apart from technical reasons, nor investigate any formal property of brave induction. In fact, temporal theories in event calculus provided as a case study in (Ray 2008) always have a single stable model, and the result of brave induction coincides with that of cautious induction in this case study.

\subsection{Further extensions and issues}

In brave induction, $B \wedge H$ has a minimal model in which an observation $O$ is satisfied. In this case, $H$ covers the positive observation $O$ under $B$. In ILP, on the other hand, negative observations as well as positive ones are also handled. Given a negative observation $N$, it is required that $H$ uncovers $N$ under $B$. This condition is logically represented as $B \wedge H \not \forall N$. Definition 2.1 is extended to handle negative observations as follows.

Definition 6.1 Let $B$ be background knowledge, $P$ a positive observation, and $N$ a negative observation. A hypothesis $H$ is a solution of brave induction if $B \wedge H$ has a minimal model $M$ such that $M \models P$ and $M \not \models N$.

Note that we are interested in minimal models in which a positive observation $P$ is true, so a negative observation $N$ is requested to be false in those minimal models. By putting $O=P \wedge \neg N$, the above definition reduces to Definition 2.1 and negative observations are handled within the framework of this paper. ${ }^{20}$

\footnotetext{
${ }^{19}$ Extended logic programs are a subclass of extended disjunctive programs such that a program contains no disjunction in the heads of rules.

${ }^{20}$ Strictly speaking, $\neg N$ requires Skolemization when a clausal theory $N$ contains variables. For detailed technique, see (Inoue 2004).
} 
Example 6.5 Consider background knowledge $B$ and an observation $O$,

$$
\begin{aligned}
& B: \text { american }(J o h n) \vee \text { english }(\text { John }), \\
& \text { american(Mary), } \\
& \text { french(Nicolas). } \\
& P \text { : speak_english(John), } \\
& N \text { : speak_english(Nicolas). }
\end{aligned}
$$

Then, putting $O=P \wedge \neg N$,

$$
\begin{aligned}
& H_{1}: \text { speak_english }(x) \leftarrow \operatorname{american}(x), \\
& H_{2}: \operatorname{speak\_ english}(x) \leftarrow \operatorname{english}(x)
\end{aligned}
$$

are two solutions of brave induction.

In this paper, we introduced induction algorithms which produce clauses or rules that define more than one predicate. The problem is known as multiple predicate learning (MPL) (De Raedt and Lavrač 1996). In MPL the order of learning different clauses affects the results of learning tasks and even the existence of solutions, especially in the presence of negative observations (De Raedt and Lavrač 1993). As discussed in Sect. 2.1, however, brave induction is not adapted for incremental learning in general. Given an observation $O$ containing multiple predicates, BRAIN computes a candidate hypothesis $H$ based on the relation $B \wedge \neg O \models \neg H \vee F$ at once. Those hypotheses are verified by checking the consistency of $B \wedge H \wedge \neg F$.

Brave induction proposed in this paper uses minimal models as a semantical basis. Due to its minimality, however, it often fails to induce useful hypotheses.

Example 6.6 John and Mary are students. John takes the courses of mathematics and physics, and Mary takes the courses of mathematics and chemistry. The situation is represented by background knowledge $B$ and the observation $O$ :

$$
\begin{aligned}
& B: \operatorname{student}(\text { John }) \wedge \operatorname{student}(\text { Mary }), \\
& O: \operatorname{math}(\text { John }) \wedge \operatorname{math}(\text { Mary }) \wedge \operatorname{physics}(\text { John }) \wedge \text { chemistry }(\text { Mary }) .
\end{aligned}
$$

In this case, the clause

$$
H: \operatorname{math}(x) \vee \operatorname{physics}(x) \vee \operatorname{chemistry}(x) \leftarrow \operatorname{student}(x)
$$

is not a solution of brave induction.

The problem of Example 6.6 is that $B \wedge H$ does not allow any student to take more than one course. In other words, disjunction is interpreted exclusively under the minimal model semantics. To allow $H$ as a solution for explaining $O$, a semantics which allows inclusive interpretations is necessary. A semantics which allows both exclusive and inclusive interpretations of disjunction in a logic program is known as the possible model semantics (Sakama and Inoue 1994). For instance, the disjunctive clause $p \vee q$ has three possibles models $\{p\},\{q\}$, and $\{p, q\}$, of which $\{p\}$ and $\{q\}$ are minimal models. Thus, the possible 
model semantics considers non-minimal models as well as minimal ones. Brave induction under the possible model semantics is defined by replacing minimal models with possible models in Definition 2.1. In Example 6.6, $B \wedge H$ has the possible model:

$$
\text { \{math(John), math(Mary), physics(John), chemistry(Mary)\}, }
$$

so that $H$ becomes a solution of brave induction for explaining $O$ under the possible model semantics. Recently, it is known that the possible model semantics characterizes the semantics of cardinality constraint rules in ASP (Marek et al. 2007). ${ }^{21}$

\section{Conclusion}

This paper introduced a logical framework of brave induction and developed algorithms in both full clausal theories and answer set programming. The utility of brave induction in problem solving was illustrated in systems biology, requirements engineering, and multiagent negotiation. Brave induction is different from the existing frameworks for induction, and provides an intermediate solution between learning from satisfiability and explanatory induction. Compared with existing frameworks, brave induction has an advantage for managing incompleteness which may arise in background knowledge, hypotheses and observations.

Brave and cautious inferences are widely used for commonsense reasoning from incomplete knowledge. In hypothetical reasoning, two different types of abduction under brave and cautious inferences are used in the literature. Since abduction and induction are both hypothetical reasoning extending background knowledge to explain observations (Flach and Kakas 2000), brave induction proposed in this paper has a right place and serves as a natural extension of brave abduction.

There are several directions for future work. From a theoretical viewpoint, this paper considered the minimal model semantics in clausal theories. Such a minimal model is defined by minimizing all predicates, but there is a notion of $(P, Z)$-minimal models in circumscription (McCarthy 1980) in which only some selected predicates $P$ are minimized and some $Z$ can be varied. Circumscriptive induction has been proposed in (Inoue and Saito 2004) by unifying descriptive and explanatory induction, so brave induction would be considered in the context of circumscriptive induction. From a computational viewpoint, the BRAIN procedure introduced in this paper is naive and needs further optimization. In particular, the introduction of inductive bias is important in practical setting. Implementing an efficient procedure for brave induction and validating its effect in practical applications are left for future work.

Acknowledgements The authors thank Yoshitaka Yamamoto for his consideration on the application to systems biology.

\section{References}

Almaas, E., Kovács, B., Vicsek, T., Oltvai, Z. N., \& Barabási, A. L. (2004). Global organization of metabolic fluxes in the bacterium Escherichia coli. Nature, 427, 839-843.

${ }^{21}$ For cardinality constraint rules, see Sect. 3.2. 
Alrajeh, D., Ray, O., Russo, A., \& Uchitel, S. (2007). Extracting requirements from scenarios with ILP. In Lecture notes in artificial intelligence: Vol. 4455. Proceedings of the 16th international conference on inductive logic programming (pp. 64-78). Berlin: Springer.

Bossu, G., \& Siegel, P. (1985). Saturation, nonmonotonic reasoning and the closed-world assumption. Artificial Intelligence, 25, 13-63.

Clark, K. L. (1978). Negation as failure. In H. Gallaire \& J. Minker (Eds.), Logic and data bases (pp. 293322). New York: Plenum.

De Raedt, L. (1997). Logical settings for concept-learning. Artificial Intelligence, 95, 187-201.

De Raedt, L., \& Dehaspe, L. (1997a). Clausal discovery. Machine Learning, 26(2-3), 99-146.

De Raedt, L., \& Dehaspe, L. (1997b). Learning from satisfiability. In Proceedings of the 9th Dutch conference on artificial intelligence (pp. 303-312).

De Raedt, L., \& Lavrač, N. (1993). The many faces of inductive logic programming. In Lecture notes in computer science: Vol. 689. Methodologies for intelligent systems, 7th international symposium (pp. 435449). Berlin: Springer.

De Raedt, L., \& Lavrač, N. (1996). Multiple predicate learning in two inductive logic programming setting. Journal of the IGPL, 4(2), 227-254.

Eiter, T., \& Gottlob, G. (1995). On the computational cost of disjunctive logic programming: propositional case. Annals of Mathematics and Artificial Intelligence, 15, 289-323.

Eiter, T., Gottlob, G., \& Leone, N. (1997). Abduction from logic programs: semantics and complexity. Theoretical Computer Science, 189, 129-177.

Flach, P. A. (1996). Rationality postulates for induction. In Proceedings of the 6th international conference on theoretical aspects of rationality and knowledge (pp. 267-281). San Mateo: Morgan Kaufmann.

Flach, P. A., \& Kakas, A. C. (2000). Abductive and inductive reasoning: background and issues. In P. A. Flach \& A. C. Kakas (Eds.), Abduction and induction-essays on their relation and integration (pp. 1-27). Dordrecht: Kluwer Academic.

Gelfond, M., \& Lifschitz, V. (1991). Classical negation in logic programs and disjunctive databases. New Generation Computing, 9, 365-385.

Gelfond, M., Przymusinska, H., \& Przymusinski, T. (1989). On the relationship between circumscription and negation as failure. Artificial Intelligence, 38, 75-94.

Helft, N. (1989). Induction as nonmonotonic inference. In Proceedings of the 1st international conference on principles of knowledge representation and reasoning (pp. 149-156). San Mateo: Morgan Kaufmann.

Inoue, K. (1992). Linear resolution for consequence finding. Artificial Intelligence, 56, 301-353.

Inoue, K. (2002). Automated abduction. In Lecture notes in artificial intelligence: Vol. 2408. Computational logic: logic programming and beyond. Essays in honour of Robert A. Kowalski, part II (pp. 311-341). Berlin: Springer.

Inoue, K. (2004). Induction as consequence finding. Machine Learning, 55, 109-135.

Inoue, K., \& Saito, H. (2004). Circumscription policies for induction. In Lecture notes in artificial intelligence: Vol. 3194. Proceedings of the 14th international conference on inductive logic programming (pp. 164-179). Berlin: Springer.

Inoue, K., \& Sakama, C. (1996). A fixpoint characterization of abductive logic programs. Journal of Logic Programming, 27(2), 107-136.

Lachiche, N. (2000). Abduction and induction from a non-monotonic reasoning perspective. In P. A. Flach \& A. C. Kakas (Eds.), Abduction and induction-essays on their relation and integration (pp. 107-116). Dordrecht: Kluwer Academic.

Lifschitz, V. (2002). Answer set programming and plan generation. Artificial Intelligence, 138, 39-54.

Marek, V. M., Niemelä, I., \& Truszczyński, M. (2007). Logic programs with monotone abstract constraint atoms. Theory and Practice of Logic Programming, 8(2), 167-199.

McCarthy, J. (1980). Circumscription-a form of nonmonotonic reasoning. Artificial Intelligence, 13, $27-39$.

McDermott, D. (1982). Nonmonotonic logic II: nonmonotonic modal theories. Journal of the ACM, 29, 3357.

Minker, J. (1982). On indefinite data bases and the closed world assumption. In Lecture notes in computer science: Vol. 138. Proceedings of the 6th international conference on automated deduction (pp. 292308). Berlin: Springer.

Muggleton, S. (1995). Inverse entailment and Progol. New Generation Computing, 13, 245-286.

Muggleton, S., \& De Raedt, R. (1994). Inductive logic programming: theory and methods. Journal of Logic Programming, 19/20, 629-679.

Niemelä, I., Simons, P., \& Soininen, T. (1999). Stable model semantics of weighted constraint rules. In Lecture notes in artificial intelligence: Vol. 1730. Proceedings of the 5th international conference on logic programming and nonmonotonic reasoning (pp. 317-331). Berlin: Springer.

Nienhuys-Cheng, S.-H., \& De Wolf, R. (1997). Lecture notes in artificial intelligence: Vol. 1228. Foundations of inductive logic programming. Berlin: Springer. 
Otero, R. P. (2001). Induction of stable models. In Lecture notes in artificial intelligence: Vol. 2157. Proceedings of the 11th international conference on inductive logic programming (pp. 193-205). Berlin: Springer.

Plotkin, G. D. (1970). A note on inductive generalization. In B. Meltzer \& D. Michie (Eds.), Machine intelligence (Vol. 5, pp. 153-163). Edinburgh: Edinburgh University Press.

Ray, O. (2008). Nonmonotonic abductive inductive learning. Journal of Applied Logic. 7(3), 329-340

Reiter, R. (1978). On closed world databases. In H. Gallaire \& J. Minker (Eds.), Logic and data bases (pp. 5576). New York: Plenum.

Sakama, C. (2000). Inverse entailment in nonmonotonic logic programs. In Lecture notes in artificial intelligence: Vol. 1866. Proceedings of the 10th international conference on inductive logic programming (pp. 209-224). Berlin: Springer.

Sakama, C. (2001). Nonmonotonic inductive logic programming. In Lecture notes in artificial intelligence: Vol. 2173. Proceedings of the 6th international conference on logic programming and nonmonotonic reasoning (pp. 62-80). Berlin: Springer.

Sakama, C. (2005). Induction from answer sets in nonmonotonic logic programs. ACM Transactions on Computational Logic, 6(2), 203-231.

Sakama, C. (2008). Inductive negotiation in answer set programming. In Lecture notes in artificial intelligence: Vol. 5397. Proceedings of the 6th international workshop on declarative agent languages and technologies (pp. 143-160). Berlin: Springer.

Sakama, C., \& Inoue, K. (1994). An alternative approach to the semantics of disjunctive logic programs and deductive databases. Journal of Automated Reasoning, 13(1), 145-172.

Sakama, C., \& Inoue, K. (2008). Brave induction. In Lecture notes in artificial intelligence: Vol. 5194. Proceedings of the 18th international conference on inductive logic programming (pp. 261-278). Berlin: Springer.

Tamaddoni-Nezhad, A., Chaleil, R., Kakas, A. C., \& Muggleton, S. (2006). Application of abductive ILP to learning metabolic network inhibition from temporal data. Machine Learning, 65, 209-230.

Yamamoto, Y., Inoue, K., \& Doncescu, A. (2009, in press). Integrating abduction and induction in biological inference using CF-Induction. In H. Lodhi \& S. Muggleton (Eds.), Wiley book series on bioinformatics. Elements of computational systems biology. New York: Wiley. 